\title{
Cristaux laser à ions de terres rares.
}

\author{
R. Moncorgé, \\ Centre Interdisciplinaire de Recherche sur les Ions et les Lasers, \\ UMR 6637 CNRS-CEA-ISMRA, Université de Caen \\ richard.moncorge@ismra.fr
}

\section{INTRODUCTION}

Bien que les diodes lasers à semiconducteurs gagnent chaque jour plus de terrain sur les autres systèmes laser grâce à leur grande efficacité de conversion électrique/optique, la diversité de leurs domaines d'émission et leur extrême compacité, leur qualité de faisceau reste quelque peu limitée et doit être souvent améliorée au prix de technologies plus ou moins complexes. De plus, il reste difficile d'en extraire de fortes puissances moyennes et leur temps de vie radiatif est trop court pour permettre le stockage d'une énergie suffisante pour produire des puissances crêtes élevées, lesquelles sont très utiles pour de nombreuses applications. Il est donc encore nécessaire de trouver des systèmes laser combinant simplicité, compacité, capacité de stockage de l'énergie et (éventuellement pour certaines utilisations) accordabilité en longueur d'onde. De ce point de vue, les matériaux dopés terres-rares eux-mêmes pompés par diodes et éventuellement associés (pour atteindre des domaines de longueur d'onde plus étendus) à différents types de matériaux non-linéaires permettent déjà de construire des systèmes lasers très attractifs et sont amenés à se développer dans le futur pour des applications très diverses.

Le but de ce chapitre n'est évidemment pas de retracer l'historique des lasers à solides dopés terres rares, car il faudrait commencer dès le début des années soixante avec les cristaux $\mathrm{CaF}_{2}: \mathrm{Sm}^{2+}$ et $\mathrm{SrF}_{2}: \mathrm{Sm}^{2+}$, ni de faire la liste des différents systèmes étudiés actuellement, car il faudrait y consacrer tout un livre. Le but est avant tout d'initier le lecteur au vocabulaire et aux différentes méthodes de caractérisation optiques utilisées par la communauté scientifique travaillant dans ce domaine. Il sera ainsi question de rendement différentiel, de puissance de pompage au seuil, de taux d'inversion minimum, de rendement d'extraction et de transferts d'énergie, puis de mesures de sections efficaces d'absorption dans les états fondamental et excités des ions, de mesures de sections efficaces d'émission stimulée, de temps de vie radiatifs et de fluorescence et de rapports de branchement. Quelques-uns de ces points seront ensuite illustrés dans une seconde partie en s'appuyant sur trois grandes familles de matériaux laser dopés terres rares, les cristaux dopés $\mathrm{Ce}^{3+}$ pour lasers accordables ou amplificateurs d'impulsions brèves dans l'ultraviolet, les matériaux dopés $\mathrm{Nd}^{3+}$ et les cristaux à basse énergie de phonons pour lasers dans le moyen-infrarouge.

\section{DÉFINITION, IDENTIFICATION ET DÉTERMINATION DES PRINCIPAUX PARAMÈTRES DE FONCTIONNEMENT}

\subsection{Généralités}

Le système laser le plus simple peut être représenté par un milieu actif inséré dans un résonateur optique à deux miroirs, le milieu actif en question étant un système atomique plus ou moins complexe 
(par exemple un ion de terre-rare dans une matrice cristalline) pouvant se ramener le plus souvent à un système dit à 3- ou à 4-niveaux. Lorsque ce milieu actif de longueur $l$ est caractérisé par le gain $g$ lorsque les pertes optiques par aller-retour de la lumière dans la cavité sont $L$ et lorsque $-\ln R$ représente la fuite à travers le miroir de sortie ( $R$ étant le coefficient de réflexion du miroir à la longueur d'onde laser), on peut alors définir la condition de seuil élémentaire :

$$
2 g l=\delta,
$$

avec $L-\ln R=\delta$.

Si la transition laser a lieu entre deux niveaux dont les densités de population (exprimés en $\mathrm{cm}^{-3}$ ) sont $N_{g}$ et $N_{e}$ (Fig. 1) et que les sections efficaces d'absorption et d'émission stimulée associées sont $\sigma_{a}$ et $\sigma_{e}$, le gain linéique $g$ s'écrit alors :

$$
g=\sigma_{e} N_{e}-\sigma_{a} N_{g},
$$

et, si les populations $N_{g}$ et $N_{e}$ sont reliés par une relation du type $N_{g}+N_{e}=N=$ constante (comme dans le cas d'un système laser à 3-niveaux où $N$ représente la densité totale en ions actifs), le gain $g$ peut également s'exprimer en fonction du taux d'inversion $\beta=\frac{N_{e}}{N}$ par :

$$
g=\left[\beta \sigma_{e}-(1-\beta) \sigma_{a}\right] N .
$$

On peut alors introduire la notion de condition de seuil minimum (ou de transparence) :

$$
g=0 \quad \Rightarrow \quad \beta=\beta_{\min }=\frac{\sigma_{a}}{\sigma_{a}+\sigma_{e}} .
$$

Il s'agit en fait du taux d'inversion minimum nécessaire pour que le gain compense les pertes par absorption dans le niveau terminal de la transition laser, ceci indépendamment des autres pertes intracavité. On vérifie ainsi aisément que $\beta_{\min }=0$ dans le cas d'un système laser 4-niveaux $\left(N_{g}=0\right)$.

Ces notions de gain et de pertes jointes aux équations d'évolution du système, celles régissant en particulier les variations spatio-temporelles des densités de population des niveaux mis en jeu dans le pompage optique et la transition laser, ainsi que de l'intensité du rayonnement émis, permettent alors de déterminer le seuil d'oscillation et le rendement de conversion énergétique du système considéré.

Ces équations d'évolution sont rapidement compliquées et seules des solutions numériques, surtout en régime impulsionnel, sont bien souvent accessibles. Une certaine procédure peut être préconisée néanmoins dans le cas d'un fonctionnement continu (régime stationnaire). Prenons pour exemple le système simplifié de la figure 1 dans lequel on a introduit le taux de pompage (nombre de photons absorbés par unité de temps et par unité de volume) $R_{p}$. Dans ce cas, la densité de population dans l'état excité $N_{e}$ et l'intensité d'émission laser $I$ circulant dans la cavité (en supposant que ces quantités sont uniformes parallèlement et perpendiculairement à l'axe du laser) obéissent aux équations d'évolution suivantes :

$$
\begin{aligned}
& \frac{\mathrm{d} N_{e}}{\mathrm{~d} t}=\varepsilon_{p} R_{p}-\frac{N_{e}}{\tau_{f}}-g \frac{I}{h \mathrm{v}_{e}}, \\
& \frac{\mathrm{d} I}{\mathrm{~d} t}=\frac{c}{n} g I-\frac{c \delta}{2 n l} I,
\end{aligned}
$$

où $c$ et $n$ sont respectivement la vitesse de la lumière et l'indice de réfraction (supposé isotrope) du milieu actif et $\tau_{f}$ désigne le temps de vie de fluorescence (émission spontanée) du niveau émetteur. 


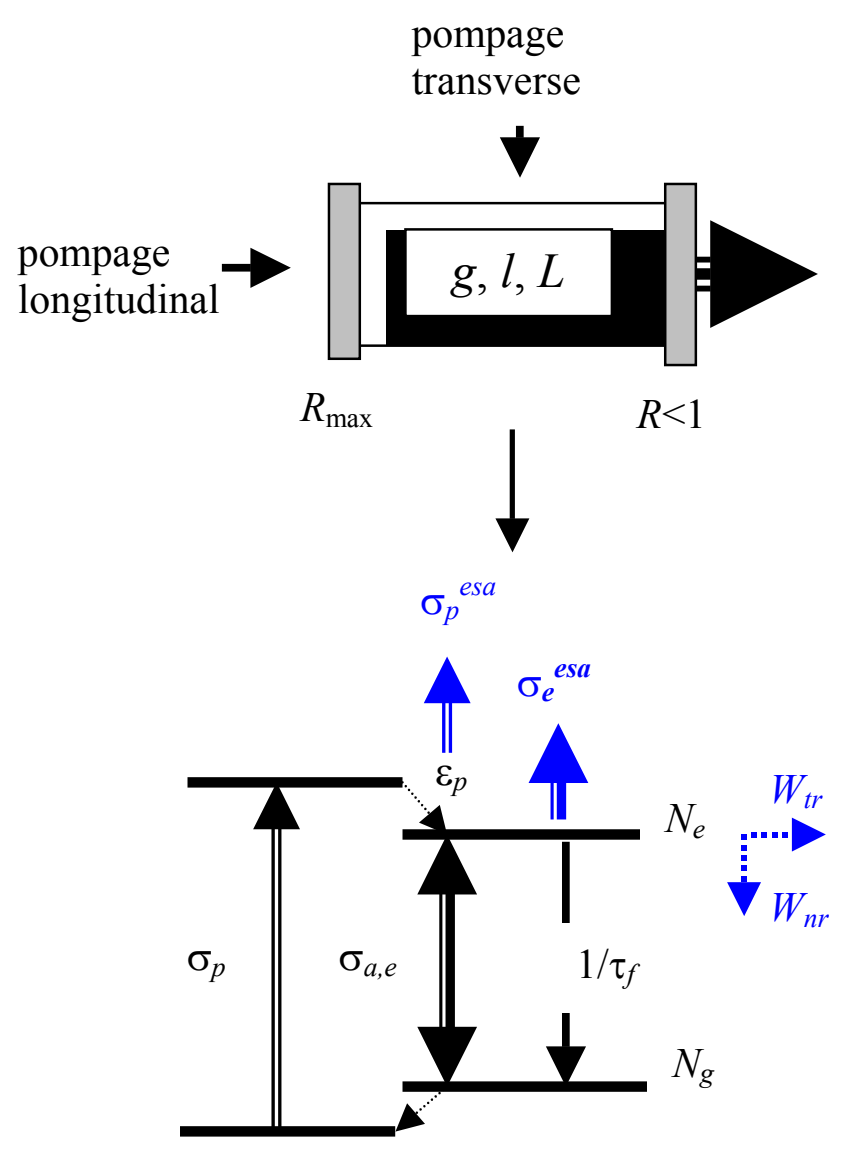

FIG. 1. Schéma simplifié de laser (résonateur optique et niveaux d'énergie) et principaux paramètres.

Connaissant $R_{p}$ et en écrivant

$$
\frac{\mathrm{d} N_{e}}{\mathrm{~d} t}=\frac{\mathrm{d} I}{\mathrm{~d} t}=0
$$

on peut d'abord trouver la puissance de pompage au seuil notée $P_{\text {seuil }}$ puis le rendement de conversion laser (pente au-delà du seuil) noté $\eta_{\text {pente}}$, sachant que la puissance à la sortie de la cavité laser sera donnée par :

$$
P_{\text {laser }}=\eta_{\text {pente }}\left(P_{\text {inc }}-P_{\text {seuil }}\right) \text {. }
$$

La puissance de pompage au seuil $P_{\text {seuil }}$ est déterminée en exprimant d'abord $R_{p}$ en fonction de la puissance de pompage incidente, laquelle est reliée à l'intensité de pompage : $I_{p}=I_{p}^{\text {seuil }}=\frac{P_{\text {seuil }}}{S}$ ( $S$ désignant la section de recouvrement des faisceaux pompe et laser dans la cavité) et de la section efficace d'absorption associée $\sigma_{p}$. On écrit alors la condition de seuil (1) en utilisant la relation ( $\left.\underline{3}\right)$ dans laquelle on aura exprimé le taux d'inversion $\beta$ en fonction de $R_{p}$ soit, d'après $(\underline{5 \mathrm{a}})$ et pour $I=0$, $\beta=\varepsilon_{p} \frac{R_{p} \tau_{f}}{N}$. Il suffit ensuite de poser $P_{\text {laser }}=$ T.I.S,$T$ représentant la transmission du miroir de sortie, et de trouver $I$ en fonction de $P_{\text {inc }}=I_{p} . S$ et de $P_{\text {seuil }}=I_{p}^{\text {seuil }} . S$ en résolvant le système d'équations ( $\underline{6})$, pour établir l'expression ( 
Ce type de procédure conduit alors (toujours en régime continu) aux expressions suivantes :

$$
\begin{aligned}
& \eta_{\text {pente }}=\eta_{q} \eta_{e} \varepsilon_{p} \eta_{p} \frac{T}{L-\ln R}, \\
& P_{\text {seuil }}=\frac{h \mathrm{v}_{l} S}{2 \eta_{q} \sigma_{e} \eta_{e} \tau_{r} \eta_{r} \varepsilon_{p} \eta_{p}}(L-\ln R),
\end{aligned}
$$

dans lesquelles nous avons fait apparaître le maximum d' "ingrédients » utiles à la caractérisation d'un système laser donné, à savoir :

- $\quad \eta_{q}=\frac{h v_{e}}{h v_{p}}$, l'efficacité de conversion quantique (parfois dénommé abusivement « défaut » quantique) mesurant la proportion d'énergie émise à la fréquence laser $v_{e}$ par rapport à celle des photons utilisés pour le pompage optique à la fréquence $v_{p}$;

- $\quad \eta_{r}=\frac{\tau_{f}}{\tau_{r}}$, le rendement quantique de fluorescence donnant le nombre de photons émis par espèce (ions) excitée, $\tau_{r}$ désignant le temps de vie radiatif du niveau émetteur ;

- $\eta_{e}=\frac{\sigma_{e}^{e f f}}{\sigma_{e}}$ où $\sigma_{e}^{e f f}=\left(\sigma_{e}+\sigma_{a}-\sigma_{e}^{e s a}\right)$ représente la section efficace d'émission stimulée effective, celle-ci pouvant être différente de $\sigma_{e}$ en cas de réabsorption du rayonnement émis dans l'état terminal de la transition laser, ceci avec la section efficace $\sigma_{a}$, ou dans l'état excité émetteur, avec la section efficace $\sigma_{e}{ }^{e s a}$;

- $\varepsilon_{p}$, l'efficacité de pompage donnant la fraction de photons absorbés se retrouvant dans le niveau émetteur ;

- et $\eta_{p}=\left[1-\exp \left(-\sigma_{p} N l\right)\right]$, le taux de pompage optique donnant la fraction de photons absorbés par photon incident.

Le facteur 2 dans l'expression (9) s'applique dans le cas d'une onde stationnaire mais doit être remplacé par 1 dans le cas d'une onde progressive (laser en anneau par exemple). D'autre part, si le faisceau de pompage optique et le faisceau laser dans la cavité sont caractérisés par les densités de modes normalisées $r_{p}(r, z)$ et $s_{0}(r, z)$ respectivement, $S$ s'écrit $S=\frac{1}{l \iint r_{p}(r, z) \cdot s_{0}(r, z) \mathrm{d} V}$. Dans le cas de faisceaux gaussiens de rayons de gorge (waists) $\omega_{p}$ et $\omega_{c}, S$ s'écrit $S=\pi\left(\omega_{p}^{2}+\omega_{c}^{2}\right) / 2$. Pour des coefficients de transmission peu importants, on peut également écrire $T \approx-\ln R$. On peut également démontrer, dans le cas d'absorption du rayonnement émis à partir de l'état terminal et/ou de l'état émetteur de la transition laser, qu'on peut remplacer le terme de pertes par aller-retour $L$, d'une part, par $L+2 \frac{\sigma_{e}^{e s a}}{\sigma_{e}} \sigma_{a} N l$ dans l'expression du rendement ( $\underline{8}$ ) et, d'autre part, par $L+2 \sigma_{a} N l$ (système laser à 3-niveaux) dans l'expression du seuil (9).

À partir de ces expressions, plusieurs remarques peuvent être faites. On constate aisément, quelle que soit la nature du système considéré, que les meilleures performances laser, i.e. les seuils les plus bas et les rendements les plus importants, seront d'abord obtenues pour des efficacités de pompage $\varepsilon_{p}$ et des sections efficaces d'absorption et d'émission stimulée effectives $\sigma_{P}$ et $\sigma_{e}^{e f f}$ importantes, en particulier pour des valeurs de sections efficaces d'émission stimulée 
« spectroscopiques » $\sigma_{e}$ élevées et dans le cas d'absorption dans l'état excité de sections efficaces $\sigma_{e}^{e s a}$ réduites. Une durée de vie $\tau_{r}$ longue et un bon rendement quantique $\eta_{r}$, donc un temps de vie de fluorescence $\tau_{f}$ long, sont d'abord souhaitables pour réduire la puissance de pompage au seuil. On sait malgré tout que de bonnes performances laser (un bon rendement en particulier) peuvent être obtenues dans le cas de durées de vie courtes et de rendements quantiques modestes [42]. D'un autre côté, un temps de vie de fluorescence $\tau_{f}$ long signifie aussi une intensité de pompage à saturation réduite. Cette grandeur notée $I_{p}^{\text {sat }}$ joue en effet un rôle très important, en particulier dans le cas de systèmes laser quasi-3 niveaux. Elle donne une mesure de l'intensité de pompage minimum notée $I_{p}{ }^{\min }$ nécessaire pour compenser les pertes par absorption dans l'état fondamental des ions actifs et pour que le milieu devienne ainsi transparent à la longueur d'onde laser. Si on reprend par exemple le schéma de pompage de la figure $\underline{1}$, le taux de pompage $R_{p}$ est relié à la puissance absorbée $P_{a b s}$ par :

$$
R_{p}=\frac{P_{a b s}}{h \mathrm{v}_{p} \cdot S . l},
$$

avec $P_{a b s}=\eta_{p} P_{i n c}$ et $P_{i n c}=I_{p} . S$.

En supposant alors $\sigma_{p} N l<<1$, ce qui est souvent vérifié, donc $\eta_{p} \approx \sigma_{p} N . l$, on peut écrire :

$$
\beta=\varepsilon_{p} \frac{R_{p} \tau_{f}}{N}=\varepsilon_{p} \frac{I_{p}}{I_{p}^{s a t}} \quad \text { avec } \quad I_{p}^{s a t}=\frac{h \mathrm{v}_{p}}{\sigma_{p} \tau_{f}},
$$

et, en supposant $\varepsilon_{p} \approx 1$ :

$$
I_{p}^{\min }=\beta_{\min } I_{p}^{s a t}
$$

$\beta_{\min }$ étant donné par la relation (4).

Ces relations peuvent être également déduites a posteriori à partir de l'expression (99) si on néglige le terme de couplage $-\ln R$, si on réduit le terme de perte $L$ au seul terme de réabsorption $2 \sigma_{a} N l$, si on néglige l'absorption dans l'état excité et si on fait de nouveau l'approximation $\eta_{p} \approx \sigma_{p} N l$. $P_{\text {seuil }}$ devient alors :

$$
P_{\text {seuil }}^{\min }=\frac{h v_{p} S}{2\left(\sigma_{a}+\sigma_{e}\right) \tau_{f} \varepsilon_{p} \sigma_{p} N l}\left(2 \sigma_{a} N l\right)=\frac{\sigma_{a}}{\sigma_{a}+\sigma_{e}} \frac{h v_{p} S}{\sigma_{p} \tau_{f}}=I_{p}^{\min } S .
$$

Un temps de vie de fluorescence $\tau_{f}$ long, donc une intensité à saturation $I_{p}^{\text {sat }}$ réduite, est également souhaitable pour un bon stockage de l'énergie, en particulier pour produire des rayonnements laser de forte puissance. Si la fraction d'ions actifs excités initialement dans le pompage optique est $\beta_{0}$, l'énergie stockée dans le volume $V=S . l$ est donnée par $E_{\text {sto }}=\beta_{0} N h v_{e} V$.

S'il s'agit par exemple d'amplification d'impulsions courtes (ou de déclenchement - Q-switch - de laser), dans lesquels l'énergie stockée est extraite très rapidement (quelques ns) comparé au temps de vie d'émission spontanée du niveau émetteur, cela signifie aussi une efficacité d'extraction :

$$
\eta_{\text {ext }}=\frac{E_{\text {out }}-E_{\text {in }}}{E_{\text {sto }}}=\frac{\beta_{0}-\beta}{\beta_{0}},
$$

$\beta$ représentant la fraction d'ions laissée dans leur état excité après amplification. Par conséquent, en principe, d'après (14), toute l'énergie stockée peut être extraite si $\beta=0$. Cependant, le rendement d'extraction maximum $\eta_{\max }$ ne sera atteint que lorsque $\beta=\beta_{\text {min }}$, donc pour :

$$
\eta_{\max }=1-\frac{\beta_{\min }}{\beta_{0}} .
$$


Cela signifie alors que la fraction maximum d'énergie stockée qui puisse être extraite est égale à la valeur de $\eta_{\max }$ obtenue pour $\beta_{0}=1$, i.e. :

$$
f_{\max }=1-\beta_{\min } .
$$

Comme cette situation ne se rencontrera uniquement lorsque tous les ions seront portés dans leur état excité, i.e. $\beta_{0}=1$, mais aussi quand la fluence du faisceau d'extraction sera nettement plus élevée que la fluence d'émission à saturation, i.e. $F_{e}^{i n}=\frac{E_{\text {in }}}{S}>>F_{e}^{s a t}=\frac{h \mathrm{v}_{e}}{\sigma_{e}+\sigma_{a}}$, donc $E_{\text {in }}>E_{\text {sat }}=F_{e}^{\text {sat }} . S$, les meilleures conditions d'extraction seront obtenues pour des sections efficaces d'émission stimulée suffisamment importantes.

Bien évidemment, dans de nombreux cas, tous les ions ne pourront pas être portés dans leur état excité et $\beta_{0}<1$. En effet, pendant le processus de pompage optique et suivant les systèmes, une partie de l'énergie d'excitation peut être perdue à cause de différents mécanismes. Les plus préjudiciables, dans le cas des matériaux dopés par des ions de terres rares ou des ions de transition, sont l'absorption dans l'état excité (ESA) à la longueur d'onde de pompage et, dans le cas de systèmes fortement concentrés en ions actifs, les transferts d'énergie entre les ions tels que ceux conduisant aux phénomènes dits d'« upconversion » et d'extinction par concentration (Fig. 1 et Fig. 2).

L'influence de l'ESA peut être examinée en déterminant, par exemple dans le cas d'un système laser quasi-3 niveaux avec $N_{e}+N_{g} \approx N$, l'évolution temporelle de la densité d'ions dans l'état excité émetteur $N_{e}$ ou la fraction d'ions excités $\beta=N_{e} / N$, en résolvant des équations du type :

$$
\frac{\partial N_{e}}{\partial t}=\left[\varepsilon_{P} \sigma_{p} N_{g}-\sigma_{p}^{e s a} N_{e}\right] \frac{I_{P}}{h \mathrm{v}_{p}}-\frac{N_{e}}{\tau_{f}},
$$

où $\sigma_{p}^{e s a}$ est la section efficace d'absorption dans l'état excité à la longueur d'onde de pompage $\lambda_{p}=\frac{c}{v_{p}}$.

En revanche, dans le cas de transferts d'énergie entre les ions, on est amené à résoudre des équations du type :

$$
\frac{\partial N_{e}}{\partial t}=\varepsilon_{p} \sigma_{p} I_{p} N-\left(\varepsilon_{p} \sigma_{p} I_{p}+\frac{1}{\tau_{f}}\right) N_{e}-2 W_{\uparrow} N_{e}^{2}
$$

où le taux d'upconversion $W_{\uparrow}$, par exemple, peut dépendre de deux sortes de transferts : des transferts directs (dits «statiques ») du type Forster-Dexter et des transferts assistés par migration (modèle de cheminement aléatoire de Burstein, par exemple), les taux de transferts associés, dans le cas d'interactions ion-ion de type dipôle-dipôle, étant proportionnels aux intégrales de recouvrement spectral :

$$
\begin{gathered}
C_{F D}=\frac{3 c}{8 \pi^{4} n^{2}} \int \sigma_{e}(\lambda) \sigma_{e}^{e s a}(\lambda) \mathrm{d} \lambda, \\
C_{m i g}=\frac{3 c}{8 \pi^{4} n^{2}} \int \sigma_{e}(\lambda) \sigma_{a}(\lambda) \mathrm{d} \lambda,
\end{gathered}
$$

lesquelles peuvent être déterminées spectroscopiquement. 


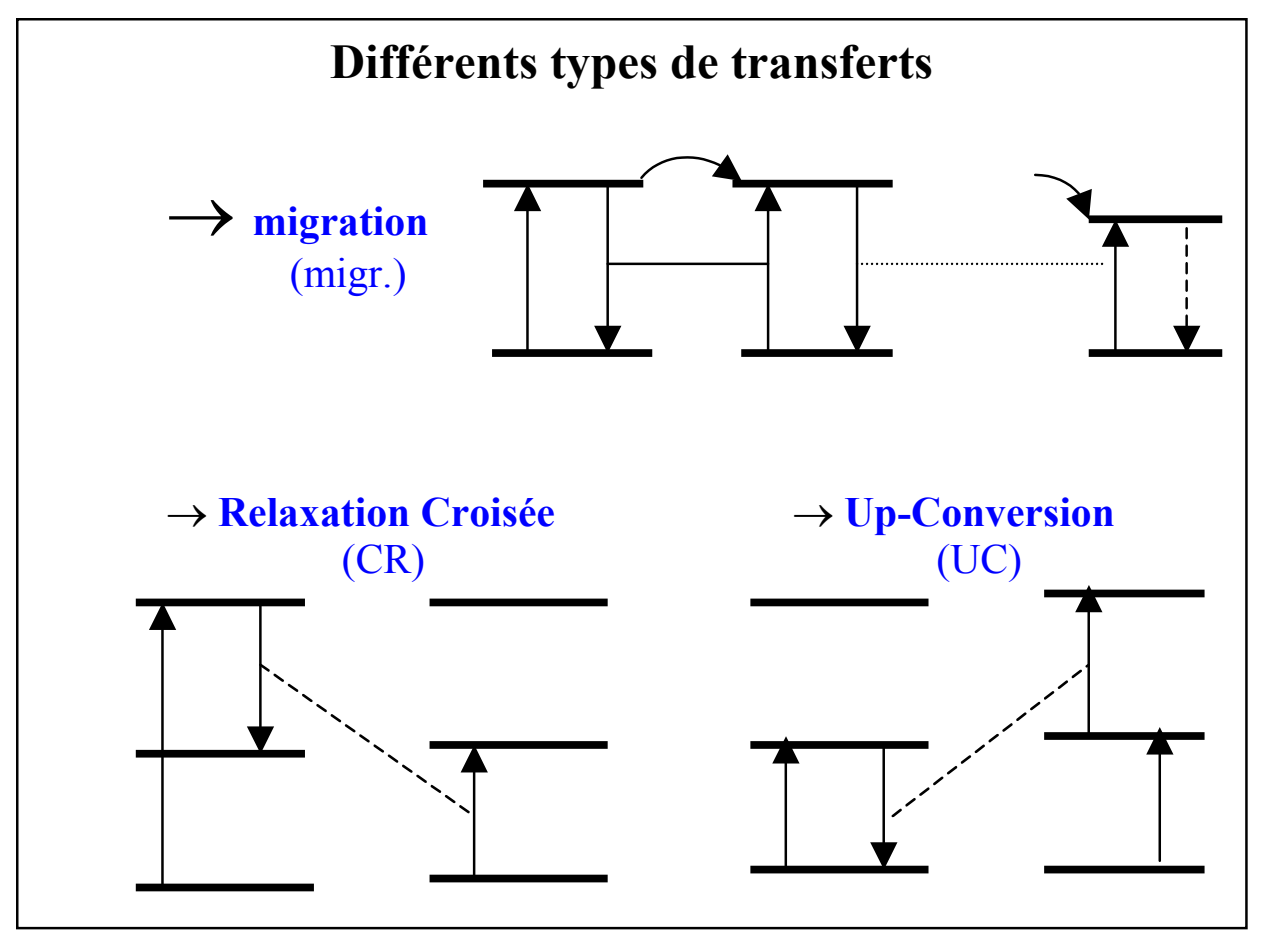

FIG. 2. Mécanismes de transferts d'énergie les plus importants intervenant dans les systèmes à ions de terres rares.

Sans entrer dans le détail, ce qui sera fait dans la suite à l'occasion d'exemples choisis, ces généralités montrent que les potentialités laser des systèmes à base d'ions de terres rares ou d'ions de transition dépendent en fait de nombreux paramètres, parmi lesquels l'intensité de pompage minimum $I_{p}^{\min }$ et la fluence à saturation à la longueur d'onde d'émission $F_{e}^{\text {sat }}$, et que la simulation et l'optimisation de leurs performances ne peuvent être réalisées sans une connaissance détaillée des propriétés spectroscopiques et dynamiques des ions actifs dans le matériau hôte considéré.

On constate ainsi que les sections efficaces d'absorption et d'émission à la longueur d'onde de pompage optique $\left(\sigma_{p}\right)$ et à la longueur d'onde laser $\left(\sigma_{a}\right.$ et $\left.\sigma_{e}\right)$ comme les temps de vie radiatif et de fluorescence $\tau_{r}$ et $\tau_{f}$ jouent des rôles très importants et que ces paramètres doivent être considérés avant tout autre. Cependant, l'ESA à la longueur d'onde de pompage optique (section efficace $\sigma_{p}^{\text {esa }}$ ) et l'ESA à la longueur d'onde laser (section efficace $\sigma_{e}{ }^{e s a}$ ), soit à l'intérieur du même ion excité, soit entre ions excités voisins échangeant (transférant) leurs énergies d'excitation, doivent être considérées quasiment en même temps. Ces absorptions dans l'état excité constituent en effet les principaux phénomènes limitateurs et il est illusoire de comparer deux systèmes laser sans considérer leurs effets.

Nous allons examiner maintenant les méthodes permettant d'obtenir des valeurs fiables pour ces différents paramètres.

\subsection{Mesure de section efficace d'absorption}

Les sections efficaces d'absorption dans l'état fondamental des ions à la longueur d'onde de pompage optique comme à la longueur d'onde laser considérées sont généralement obtenues sans difficulté. Il suffit d'enregistrer un spectre de densité optique ou de transmission et les sections efficaces $\sigma_{p(a)}$ sont immédiatement déduites des coefficients d'absorption mesurés $\alpha_{p(a)}$ en utilisant la relation $\alpha_{p(a)}=\sigma_{p(a)} N$. Cependant, la concentration totale en ions actifs $N$ doit être connue ou estimée avec 
précision, ce qui n'est pas toujours évident. Elle peut être estimée avec une assez bonne précision à partir de la composition de départ du matériau si le coefficient de ségrégation des ions dopants dans le matériau en question est lui-même bien défini. Elle peut être mesurée chimiquement, à l'aide de méthodes telle que la méthode ICP (Induction-Coupled Plasma). Elle peut être obtenue par comparaison avec des spectres d'absorption trouvés dans la litérature, à condition que ces spectres soient eux-mêmes calibrés avec précaution.

Une autre méthode consiste à enregistrer, à une longueur d'onde particulière, la transmission $T$ d'un faisceau laser à impulsions passant à travers l'échantillon à tester en fonction de son intensité ou de sa fluence, et d'ajuster les données, quand la durée de l'impulsion laser vérifie $\tau_{p}<<\tau_{f}$ (absorbant saturable lent), avec une expression telle que l'expression de Frantz-Nodvik modifiée [20], i.e. :

$$
T=\frac{F_{p}^{\text {out }}}{F_{p}^{\text {in }}}=\frac{F_{p}^{\text {sat }}}{F_{p}^{\text {in }}} \ln \left\{1+\left[\exp \left(\frac{F_{p}^{\text {in }}}{F_{p}^{\text {sat }}}\right)-1\right] T_{0}\right\},
$$

avec $F_{p}=\int_{0}^{\tau_{p}} I_{p}(t) \mathrm{d} t, F_{p}^{s a t}=\frac{h \cup_{p}}{\sigma_{p}}$ et $T_{0}=\exp \left(-\sigma_{p} N l\right)$ ou, en présence d'ESA à la longueur d'onde de pompage et toujours quand $\tau_{p}<<\tau_{f}$ est vérifié, en intégrant une expression du type [30] :

$$
\frac{d F_{p}}{d z}=-\left(\sigma_{p}-\sigma_{p}^{e s a}\right) N F_{p}^{s a t}\left[1-\exp \left(-\frac{F_{p}}{F_{p}^{s a t}}\right)\right]-\left(\sigma_{p}^{e s a} N\right) F_{p},
$$

une procédure ne requérant que trois paramètres d'ajustement, $\sigma_{p}$, $\sigma_{p}^{e s a}$ et $N$, et qui donnent souvent de bons résultats. Aux faibles fluences de pompage $F_{p}<<F_{p}^{\text {sat }}$, après intégration sur la longueur $l \mathrm{de}$ l'échantillon, on obtient $T \cong T_{0}$. Par contre, aux fortes fluences, l'expression (21) donne [40] :

avec

$$
\begin{gathered}
T=T_{\text {sat }}+\left(1-\frac{\sigma_{p}}{\sigma_{p}^{e s a}}\right)\left(1-T_{\text {sat }}\right) \frac{F_{p}^{\text {sat }}}{F_{p}^{\text {in }}}, \\
T_{\text {sat }}=T_{0}^{\gamma} \text { et } \gamma=\frac{\sigma_{p}^{\text {esa }}}{\sigma_{p}},
\end{gathered}
$$

et $T$ tend asymptotiquement vers $T_{\text {sat }}$.

Il ne faut pas oublier cependant que ces expressions simplifiées ne s'appliquent bien que dans le cas d'impulsions laser à profil carré («Top-Hat»). Une procédure numérique plus compliquée est généralement nécessaire dans le cas de profils spatio-temporels quelconques [65].

\subsection{Mesure de section efficace d'émission stimulée}

Deux types de méthodes peuvent être utilisées pour déterminer une section efficace d'émission stimulée :

1) Les méthodes spectroscopiques de McCumber, dite aussi de « réciprocité » RM $[\underline{48}, \underline{1}, \underline{58}]$, et de Fuchtbauer-Ladenburg (FL) $[\underline{28}, \underline{52}]$ qui reposent néanmoins sur une parfaite connaissance de la structure énergétique de l'ion émetteur et des mesures de spectres d'absorption et d'émission, respectivement.

2) Les méthodes «laser », i.e. les mesures de «gain à petit signal» [57], de saturation de gain [45], de seuil et de rendement. 


\section{Méthodes spectroscopiques}

Si on retourne à la définition des fameux coefficients d'Einstein (voir Appendice A), les coefficients d'absorption $\alpha(v)$ et de gain $g(v)$ à la fréquence $v$ entre deux niveaux atomiques $i$ et $j$ de dégénérescence $g_{i}$ et $g_{j}$ respectivement, le dernier étant le plus haut, sont donnés par la relation :

$$
\begin{gathered}
g(\mathrm{v})=-\alpha(\mathrm{v})=\sigma\left(g_{i} N_{j}-g_{j} N_{i}\right), \\
\text { avec } \sigma=\frac{c^{2}}{8 \pi \mathrm{v}^{2}} \frac{A_{j i}}{g_{i}} \Phi(\mathrm{v}) \quad \text { et } \quad \sigma=\frac{\sigma_{j i}}{g_{i}}=\frac{\sigma_{i j}}{g_{j}} .
\end{gathered}
$$

En supposant que $i$ soit un des niveaux Stark d'un état bas $g$ (état fondamental par exemple) d'un ion de terre rare donné et $j$ un niveau Stark d'un de ses états excités $e$, les sections efficaces d'absorption et d'émission stimulée «spectroscopiques » $\sigma_{a}(\lambda)$ et $\sigma_{e}(\lambda)$ à la longueur $\lambda$ entre ces deux niveaux (Fig. 3) sont obtenues, à une température $T$, en sommant les sections efficaces individuelles $\sigma_{i j}$ et $\sigma_{j i}$ pondérées par les facteurs d'occupation thermique $f_{i}=\frac{N_{i}}{N_{g}}$ et $f_{j}=\frac{N_{j}}{N_{e}}$ des niveaux $i$ et $j$ respectivement, suivant les expressions :

$$
\sigma_{a}(\lambda)=\sum_{i, j} f_{i} \sigma_{i j}(\lambda) \text { et } \sigma_{e}(\lambda)=\sum_{i, j} f_{j} \sigma_{j i}(\lambda),
$$

avec

$$
f_{i(j)}=\frac{g_{i(j)} \exp \left(-E_{i(j)} / k T\right)}{Z_{g(e)}}
$$

$E_{i(j)}$ désignant les énergies des niveaux $i$ et $j$ mesurées par rapport aux niveaux Stark les plus bas de chaque état multiplet fondamental et excité $g$ et $e$,

et

$$
Z_{g(e)}=\sum_{i(j)} g_{i(j)} \exp \left(-E_{i(j)} / k T\right),
$$

les fonctions de partition de ces multiplets.

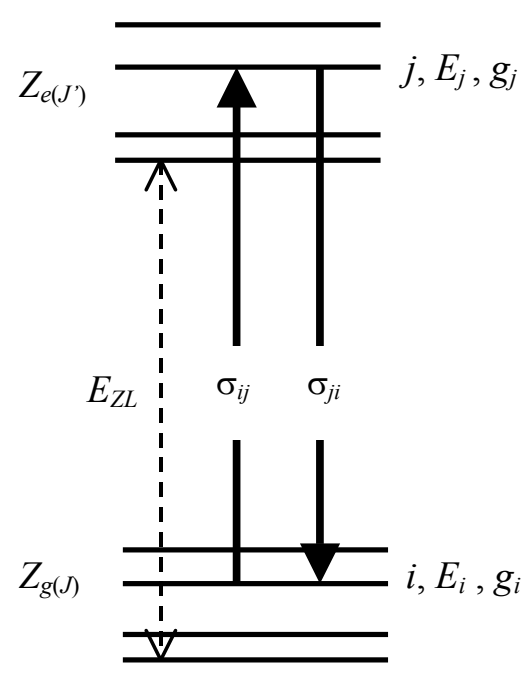

FIG. 3. Schéma de niveaux d'énergie habituels d'un ion de terre-rare. 
À ce niveau, plusieurs remarques peuvent être faites :

1) Si un seul couple de sous-niveaux Stark $i$ et $j$ correspond à une transition à la longueur d'onde $\lambda$ considérée, alors :

$$
\sigma_{a}(\lambda)=f_{i} \sigma_{i j}(\lambda) \quad \text { et } \quad \sigma_{e}(\lambda)=f_{j} \sigma_{j i}(\lambda),
$$

ce qui donne, d'après $(\underline{25}): \quad \frac{\sigma_{e}}{\sigma_{a}}=\frac{f_{j}}{f_{i}} \frac{g_{i}}{g_{j}}$.

2) Quand toutes les positions des sous-niveaux Stark d'un multiplet donné ne peuvent pas être déterminées expérimentalement (en particulier dans le cas de système amorphe), les fonctions de partition associées peuvent être déterminées soit en utilisant les positions théoriques des niveaux résultant de calculs plus ou moins sophistiqués, soit en utilisant la relation approchée :

$$
Z_{J}=\frac{N_{\text {prévus }}^{J}}{N_{\text {observés }}^{J}},
$$

où le nombre de sous-niveaux prévus pour un multiplet de nombre quantique $J$, dépend de l'éclatement de ce multiplet sous l'effet du champ cristallin ( $\underline{\text { Appendice B }}$ ), sachant que ce nombre $N_{\text {prévus }}^{J}$ ne peut être supérieur à $2 J+1$ quand $J$ est pair et $J+1 / 2$ quand $J$ est impair.

3) $\beta_{\min }$, le taux d'inversion minimum nécessaire pour atteindre la transparence du milieu (en supposant que $N_{g}+N_{e}=N$ ), est donné par :

$$
\begin{aligned}
& \alpha=\sigma_{a} N_{g}-\sigma_{e} N_{e}=\sigma\left(g_{j} f_{i} N_{g}-g_{i} f_{j} N_{e}\right)=\sigma\left[g_{j} f_{i} N-\left(g_{j} f_{i}+g_{i} f_{j}\right) N_{e}\right]=0, \\
& \text { donc : } \quad \beta=\beta_{\min }=\frac{N_{e}}{N}=\frac{\sigma_{a}}{\sigma_{a}+\sigma_{e}}=\frac{g_{j} f_{i}}{g_{j} f_{i}+g_{i} f_{j}} \\
& \text { ou, quand } g_{i}=g_{j}: \quad \beta=\beta_{\text {min }}=\frac{f_{i}}{f_{i}+f_{j}}
\end{aligned}
$$

Si on introduit l'énergie de séparation $E_{Z L}(Z L$ pour «Zero-Line») entre les composantes Stark les plus basses de chaque multiplet ( $\underline{\text { Fig. 3 }}$ ) et si on écrit $E_{j}-E_{i}=\frac{h c}{\lambda}-E_{Z L}$, on obtient la relation de « réciprocité » bien connue :

$$
\sigma_{e}(\lambda)=\sigma_{a}(\lambda) \frac{Z_{g}}{Z_{e}} \exp \left[\left(E_{Z L}-\frac{h c}{\lambda}\right) / k T\right] .
$$

À partir de cette relation, le spectre de section efficace d'émission stimulée $\sigma_{e}(\lambda)$ peut être aisément construit à partir du spectre de section efficace d'absorption $\sigma_{a}(\lambda)$, en tout cas quand ce dernier n'est pas trop bruité (en particulier dans la région d'émission de plus grande longueur d'onde). Cette méthode très pratique a été souvent utilisée ces dernières années dans le cas des transitions laser quasi3 niveaux ${ }^{4} \mathrm{I}_{9 / 2} \leftrightarrow{ }^{4} \mathrm{~F}_{3 / 2},{ }^{4} \mathrm{I}_{15 / 2} \leftrightarrow{ }^{4} \mathrm{I}_{13 / 2},{ }^{3} \mathrm{H}_{4} \leftrightarrow{ }^{3} \mathrm{~F}_{4},{ }^{5} \mathrm{I}_{8} \leftrightarrow{ }^{5} \mathrm{I}_{7}$ et ${ }^{2} \mathrm{~F}_{7 / 2} \leftrightarrow{ }^{2} \mathrm{~F}_{5 / 2}$ des ions $\mathrm{Nd}^{3+}, \mathrm{Er}^{3+}$, $\mathrm{Tm}^{3+}, \mathrm{Ho}^{3+}$ et $\mathrm{Yb}^{3+}$ autour de $0,94,1,55,1,92$ et 1,03 $\mu \mathrm{m}$, respectivement. Elle a été également utilisée dans le cas de transitions laser 4-niveaux [68] pour déterminer, par exemple, la section efficace d'émission stimulée du Nd:YAG à $1,064 \mu \mathrm{m}$. Dans ce cas, la transition considérée $\left({ }^{4} \mathrm{I}_{11 / 2} \leftrightarrow{ }^{4} \mathrm{~F}_{3 / 2}\right)$ a lieu entre le niveau métastable ${ }^{4} \mathrm{~F}_{3 / 2}$ et le multiplet ${ }^{4} \mathrm{I}_{11 / 2}$ situé à environ $2000 \mathrm{~cm}^{-1}$ au-dessus du niveau 
fondamental, et des coefficients d'absorption très faibles $\left(\alpha \cong 10^{-3}-10^{-4} \mathrm{~cm}^{-1}\right)$ devaient être mesurées.

Bien que la méthode de réciprocité (RM) soit la plus fiable, la méthode de FuchtbauerLadenburg » (FL) est également très pratique, au moins pour confirmer les résultats donnés par la première. L'équation correspondante s'écrit :

$$
\sigma_{e}^{p}(\lambda)=\frac{3 \lambda^{5} \beta_{r}}{8 \pi c \tau_{r} n_{p}^{2}} \frac{I_{e}^{p}(\lambda)}{\int \sum_{p} \lambda I_{e}^{p}(\lambda) d \lambda},
$$

où $I_{e}^{p}$ représente l'intensité d'émission mesurée en polarisation $p, n_{p}$ l'indice de réfraction du matériau dans le domaine de longueurs d'onde considéré et $\beta_{r}$ le rapport d'embranchement radiatif de la transition dans le cas d'émissions à partir d'un niveau métastable donné vers plusieurs multiplets $\left({ }^{4} \mathrm{~F}_{3 / 2}\right.$ vers ${ }^{4} \mathrm{I}_{9 / 2},{ }^{4} \mathrm{I}_{11 / 2},{ }^{4} \mathrm{I}_{3 / 2}$ et ${ }^{4} \mathrm{I}_{15 / 2}$ par exemple dans le cas de l'ion $\left.\mathrm{Nd}^{3+}\right)$.

Bien que l'expression ( $\underline{35}$ ) ne nécessite aucune description détaillée des échelles de niveaux d'énergie des ions actifs, elle nécessite cependant une connaissance très précise du temps de vie radiatif $\tau_{r}$ et du rapport d'embranchement $\beta_{r}$, ce qui n'est pas toujours évident. La façon la plus directe de déterminer $\tau_{r}$ est de mesurer le temps de vie de fluorescence $\tau_{f}$ correspondant et de supposer $\tau_{r} \approx \tau_{f}$, ce qui s'applique quand le rendement quantique d'émission $\eta_{r}=\frac{\tau_{f}}{\tau_{r}} \approx 1$. Malheureusement, le temps de vie de fluorescence $\tau_{f}$ mesuré peut varier et être raccourci ou allongé par rapport à $\tau_{r}$, d'abord à cause de relaxations non-radiatives multiphonons entre multiplets adjacents (Appendice C), mais aussi à cause de transferts d'énergie très divers : 1) vers des impuretés ou des défauts de réseau, 2) vers des ions actifs adjacents du même type que l'ion excité émetteur, soit sous forme non-radiative (cas de matériaux fortement concentrés en ions dopants) soit sous forme radiative (phénomène de réabsorption ou de piègeage radiatif dans les systèmes quasi-3 niveaux). La détermination expérimentale précise du rapport $\beta_{r}$ passe quant à elle par la calibration de toutes les bandes d'émission associées aux diverses transitions issues d'un niveau émetteur donné, ce qui n'est pas simple lorqu'il s'agit de domaines spectraux très différents et qu'il faut changer à la fois de détecteurs et de spectromètres d'analyse.

Plusieurs remarques peuvent être encore faites concernant l'expression ( $\underline{35})$.

1) Dans le cas d'un système uniaxe d'axe optique noté (ao), l'état de polarisation $p$ est :

- $\pi$ lorsque le vecteur champ électrique $\vec{E} / /$ ao et que le vecteur de propagation $\vec{k} \perp$ ao ;

- $\sigma$ quand $\vec{E} \perp$ ao et $\vec{k} \perp$ ao ;

- $\alpha$ quand $\vec{E} \perp$ ao et $\vec{k} / /$ ao.

Par conséquent, dans le cas de transitions dipolaires électriques, les spectres $\alpha$ et $\sigma$ sont équivalents tandis que dans le cas de transitions dipolaires magnétiques, les spectres $\alpha$ sont équivalents aux spectres $\pi$.

2) Dans le cas d'un système uniaxe on pourra donc écrire :

$$
\begin{aligned}
& \sum_{q} I^{p}=I^{\pi}+2 I^{\sigma}, \\
& \frac{\beta_{r}}{\tau_{r}}=A_{r}=\frac{1}{3} A_{\pi}+\frac{2}{3} A_{\sigma},
\end{aligned}
$$


et si l'émission est fortement polarisée $\pi$, i.e. $A_{\pi}>>A_{\sigma}$, alors, d'après ( $\left.\underline{35}\right)$, on constate que le produit $\sigma_{e}{ }^{\pi} \cdot \tau_{r}$ sera approximativement trois fois plus grand que dans le cas d'une émission complètement dépolarisée. Cela signifie qu'il est intéressant de rechercher des systèmes uniaxes avec de grandes sections efficaces en polarisation $\pi$. C'est le cas, par exemple, des cristaux de vanadates $\mathrm{YVO}_{4}, \mathrm{GdVO}_{4}$ et $\mathrm{LuVO}_{4}$ dopés $\mathrm{Nd}^{3+}$ [47] pour lesquels on a enregistré des sections efficaces record à $1,065 \mu \mathrm{m}$, en polarisation $\pi$ et à température ambiante de l'ordre de $13,5 \cdot 10^{-19} \mathrm{~cm}^{2}$.

3) Dans le cas de cristaux biaxes, les spectres d'absorption et d'émission sont habituellement enregistrés avec la lumière polarisée selon chacun des trois axes $X, Y$ et $Z$ de l'ellipsoïde des indices. L'expression (35) peut être alors utilisée avec $p=X, Y$ et $Z$ et, à partir des valeurs $\sigma_{e, X}, \sigma_{e, Y}$ et $\sigma_{e, Z}$, la section efficace peut être calculée pour n'importe quelle direction de polarisation. C'est très important par exemple dans le cas de cristaux non-linéaires pour lesquels la direction de propagation de la lumière et sa polarisation doivent obéir à certaines conditions d' " accord de phase» pour produire un rayonnement particulier (doublage ou mélange de fréquences). Si la direction de polarisation souhaitée fait l'angle $\theta$ avec l'axe $Z$ et si sa projection dans le plan $X Y$ fait l'angle $\phi$ avec l'axe $X$, la section efficace d'émission stimulée peut-être calculée à l'aide de l'expression [46] :

$$
\sigma(\theta, \varphi)=\frac{\sigma_{X} \sigma_{Y} \sigma_{Z}}{\left[\sigma_{X}^{2} \sigma_{Y}^{2} \cos ^{2}(\theta)+\sigma_{Y}^{2} \sigma_{Z}^{2} \sin ^{2}(\theta)+\sigma_{X}^{2} \sigma_{Z}^{2} \sin ^{2}(\varphi) \sin ^{2}(\theta)\right]^{1 / 2}}
$$

4) L'expression ( $\underline{35}$ ) indique également que la section efficace d'émission $\sigma_{e}$ est approximativement inversement proportionnelle au produit de la largeur spectrale d'émission par le temps de vie radiatif et le carré de l'indice de réfraction du matériau. Cela signifie d'abord que les grandes sections efficaces seront trouvées, en général, dans les cristaux monosites à élargissement spectral homogène (lequel est habituellement étroit) et dans les matériaux à faible indice de réfraction tel que les fluorures. Cependant, comme on le verra plus loin dans le tableau 2 , ceci ne peut pas être considéré comme une règle. Le second point concerne le seuil d'oscillation laser, l'énergie stockée et le rendement d'extraction qui, d'après le paragraphe précédent, devraient être d'autant meilleurs que le produit $\sigma_{e} \cdot \tau_{f}$, donc $\sigma_{e} \cdot \tau_{r}$, est grand. Cela signifie qu'un compromis doit être trouvé, ce compromis n'étant pas indépendant du mode de fonctionnement laser considéré. Dans le cas de systèmes laser de fortes puissances, par exemple, et dans une certaine limite, un temps de vie long est généralement préférable à une grande section efficace d'émission stimulée ; en effet, plus d'énergie peut être stockée tout en réduisant les pertes par amplification d'émission spontanée (ASE) [41]. C'est pour cette raison qu'on préfère utiliser un barreau de YAP:Nd d'axe parallèle à son axe cristallographique $c$ pour un régime déclenché qu'un barreau orienté suivant l'axe $b$, préférable en régime continu, bien que cette deuxième configuration corresponde à une section efficace d'émission stimulée pour E//c plus importante $[\underline{3}, \underline{51]}$.

\section{Méthodes "laser »}

Ces méthodes nécessitent bien souvent des montages appropriés, en particulier des lasers qui ne sont pas toujours très standards. En outre, elles ne sont pas toujours très fiables car les résultats des mesures dépendent non seulement des conditions expérimentales et de la qualité optique des échantillons mais aussi du degré de complexité du modèle utilisé pour analyser les données. D'un autre côté, elles restent intéressantes car elles constituent souvent l'étape ultime de vérification des divers paramètres spectroscopiques.

Les mesures de « gain à petit-signal » consistent à mesurer l'amplification d'un faisceau laser « sonde » à une longueur d'onde particulière $\lambda$ au passage d'un milieu actif, lequel est optiquement pompé par un autre laser (laser «pompe ») à la fréquence $v_{p}$. Si on suppose, par exemple, que les 
faisceaux pompe et sonde se recouvrent dans une région de section $S$ et de longueur $l$ dans la direction $z$, le gain à petit signal sera donné, avec les notations utilisées dans le paragraphe précédent, par :

$$
G=\frac{F_{e}^{\text {out }}}{F_{e}^{\text {in }}}=\exp \int_{0}^{l}\left[\beta\left(\sigma_{e}-\sigma_{e s a}\right)-(1-\beta) \sigma_{a}\right] N \mathrm{~d} z,
$$

$\operatorname{avec} \beta(z)=\frac{N_{e}(z)}{N}$

Dans le cas d'un système laser 4-niveaux, donc pour $\sigma_{a}=0$, l'expression (푸) devient :

$$
G=\frac{F_{e}^{\text {out }}}{F_{e}^{\text {in }}}=\exp \int_{0}^{L} \beta\left(\sigma_{e}-\sigma_{e s a}\right) N \mathrm{~d} z \cong \exp \left(\sigma_{e}-\sigma_{e s a}\right) \frac{\varepsilon_{p} I_{p}}{I_{p}^{\text {sat }}} N l,
$$

soit

$$
G \cong \exp \left(\sigma_{e}-\sigma_{e s a}\right) \frac{\varepsilon_{p} P_{a b s} \tau_{f}}{S h v_{p}} .
$$

De telles mesures de gain peuvent donc données directement la section efficace d'émission stimulée effective $\sigma_{e}^{e f f}=\sigma_{e}-\sigma_{e}^{e s a}$.

Une autre méthode consiste à enregistrer la « saturation du gain » en fonction de l'énergie $E_{\text {in }}$ ou de la fluence $F_{e}^{\text {in }}$ d'un faisceau sonde à impulsions, en utilisant une expression analogue à l'expression (20)$)$, soit :

$$
G=\frac{E_{\text {out }}}{E_{\text {in }}}=\frac{E_{\text {sat }}}{E_{\text {in }}} \ln \left\{1+\left[\exp \left(\frac{E_{\text {in }}}{E_{\text {sat }}}\right)-1\right] G_{0}\right\},
$$

où $G_{0}=\exp \left(\beta_{0} \sigma_{e} N l\right)=\exp \left(\frac{E_{\text {sto }}}{E_{\text {sat }}}\right)$ est le gain à petit signal.

Une troisième méthode consiste à utiliser les mesure de seuils et de rendements laser. Cependant, comme le montre les expressions ( $\underline{8})$ et ( $\underline{9})$, les pertes par aller-retour, le temps de vie radiatif du niveau émetteur et plusieurs autres facteurs de rendements doivent être évalués, ce qui peut être difficile à réaliser de manière suffisamment précise. De plus, comme plus haut, l'ESA peut perturber les résultats. Une technique a été proposée récemment [55] pour contourner quelques-unes de ces difficultés quand la puissance de pompage au seuil $P_{\text {seuil }}$ et l'efficacité laser $\eta_{\text {pente }}$ ont été mesurées pour plusieurs transmissions de miroirs de sortie $T \cong-\ln R$. En effet, les expressions ( $\underline{8}$ ) et (9) peuvent être combinées pour donner :

$$
P_{\text {seuil }} . \eta_{\text {pente }}=\frac{h \mathrm{v}_{l} S}{2 \sigma_{e} \tau_{r} \eta_{r}} T
$$

et la section efficace d'émission «spectroscopique » $\sigma_{e}$ est alors simplement déduite de la pente de la droite donnant $P_{\text {seuil }} . \eta_{\text {pente }}$ en fonction de $T$, ceci à condition que $S$ et $\tau_{f}=\tau_{r} . \eta_{r}$ soient connus avec une bonne précision.

On peut également remarquer que le produit $P_{\text {seuil }} . \eta_{\text {pente }}$ est directement donné par l'intersection de la droite de rendement laser figurant la puissance laser en fonction de la puissance de pompage incidente avec l'axe des ordonnées, i.e. pour $P_{\text {inc }}=0$, puisqu'alors $P_{\text {Laser }}=-P_{\text {seuil }} \cdot \eta_{\text {pente }}$. 


\subsection{Mesure de section efficace dans l'état excité}

Les mesures de section efficace d'absorption dans les états excités des ions de terres-rares peuvent se révéler très délicates. En effet, les sections efficaces mises en jeu sont relativement faibles. Les transitions optiques concernées peuvent coïncider avec des transitions optiques à partir de l'état fondamental ou d'autre états excités. Le nombre d'ions qu'il est possible de porter dans l'état excité considéré peut être insuffisant pour obtenir un signal sur bruit significatif.

Les méthodes expérimentales utilisées reposent toutes sur des techniques "pompe-sonde» mais celle qui a donnée jusqu'à présent les meilleurs résultats est la technique de double-modulation utilisant des sources de lumière à fonctionnement continu. La source de pompage optique est un laser tel un laser à argon ionisé, un laser Titane-saphir, voire un laser à centres coloré avec une puissance de l'ordre du watt. La sonde peut être un autre laser accordable du même type, si on ne veut sonder qu'un domaine spectral restreint, ou une lampe à large bande telle une lampe à iode à filament de tungstène dont on sélectionne la longueur d'onde à l'aide d'un monochromateur. Chacun des faisceaux modulés à des fréquences très différentes traverse l'échantillon à étudier et le signal de sonde transmis est analysé à travers deux détections synchrones disposées en série. On enregistre alors un signal de la forme [39] :

$$
\frac{\Delta I}{I}(\lambda)=A N^{*} l\left[\sigma_{a}(\lambda)+\sigma_{e}(\lambda)-\sigma_{e s a}(\lambda)\right],
$$

où $\Delta I$ représente la variation d'intensité du signal de sonde $I$ transmis par l'échantillon lorsqu'il est soumis ou non au faisceau de pompage optique. $A$ est une sorte de fonction d'appareil qui prend en compte les calibres et les constantes de temps des détections synchrones. $N^{*}$ est le nombre d'ions portés dans l'état excité et $l$ la longueur de l'échantillon. Le spectre de section efficace d'absorption dans l'état excité $\sigma_{e s a}(\lambda)$ peut être alors construit connaissant $\sigma_{a}$, $\sigma_{e}$ (à l'aide d'une des méthodes décrites précédemment), $A$ et $N^{*}$, ce dernier pouvant être déterminé soit à partir des conditions expérimentales, soit à partir des spectres dans les domaines de longueurs d'onde où l'on sait que $\sigma_{e s a}$ est négligeable.

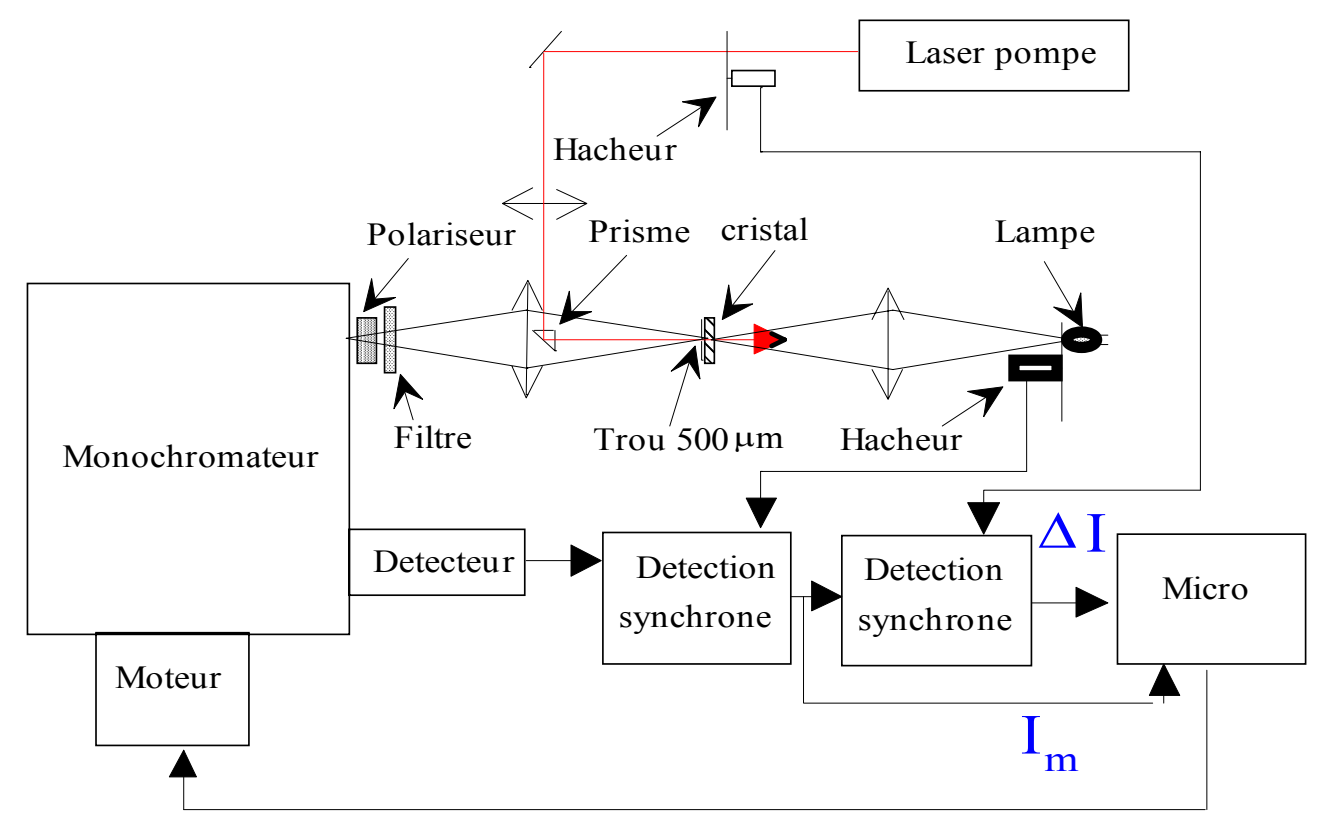

FIG. 4. Montage à double détection synchrone utilisé au CIRIL-MIL de Caen pour enregistrer les spectres d'absorption dans les états excités des matériaux dopés par des ions de terres rares. 
Il s'agit d'une technique fiable mais délicate à mettre en œuvre, ce qui explique que peu de laboratoires (Institut Fur Laser Physik d'Hamburg, CIRIL-MIL de Caen) la maîtrisent vraiment. Un schéma de principe est reportée sur la figure $\underline{4}$.

\subsection{Détermination des temps de vie et rapports d'embranchement radiatifs}

Rappelons tout d'abord que les transitions optiques entre niveaux ${ }^{2 \mathrm{~S}+1} \mathrm{~L}_{\mathrm{J}}$ des ions de terres rares obéissent aux règles de sélection suivantes :

$$
\begin{aligned}
& \Delta \mathrm{L}= \pm 1, \Delta \mathrm{S}=0,|\Delta \mathrm{L}|,|\Delta \mathrm{J}| \leq 6 \text { pour les transitions dipolaires électriques (ED), } \\
& \Delta \mathrm{L}=\Delta \mathrm{S}=0, \Delta \mathrm{J}=0 \pm 1 \text { (mais pas } 0 \leftrightarrow 0 \text { ) pour les transitions dipolaires magnétiques (MD). }
\end{aligned}
$$

Or, dans le cadre du formalisme de Judd-Ofelt, les sections efficaces intégrées des transitions permises dipolaires électriques peuvent s'écrire :

$$
S_{E D}^{\text {calc }}\left(J \rightarrow J^{\prime}\right)=\sum_{t=2,4,6} \Omega_{t}\left|\left\langle\Psi_{j} / / U^{(t)} / / \Psi_{J^{\prime}}\right\rangle\right|^{2} .
$$

Les $<\mathrm{U}^{(t)}>$ sont des éléments de matrice tabulés $[\underline{9}, \underline{10}]$, et les $\Omega_{\mathrm{t}}$ les paramètres d'intensité (Judd-Ofelt) déterminés à partir des spectres de sections efficaces d'absorption mesurés, $\sigma_{a}(\lambda)$, à l'aide des expressions $[\underline{69}, \underline{8}]$ :

$$
S_{E D}^{m e s}\left(J \rightarrow J^{\prime}\right)=\frac{9 n}{\left(n^{2}+2\right)^{2}}\left[\frac{3 h(2 J+1)}{8 \pi^{3} e^{2} \bar{\lambda}} \int \sigma_{a}(\lambda) \mathrm{d} \lambda-n S_{M D}\left(J \rightarrow J^{\prime}\right)\right],
$$

avec

$$
S_{M D}\left(J \rightarrow J^{\prime}\right)=\left(\frac{h}{4 \pi m c}\right)^{2}\left|\left\langle\Psi_{J} / / L+2 S / / \Psi_{J^{\prime}}\right\rangle\right|^{2}
$$

et en écrivant: $\Omega=\left(\begin{array}{l}\Omega_{2} \\ \Omega_{4} \\ \Omega_{6}\end{array}\right)=\left(A^{+} A\right)^{-1} A^{+} S_{E D}^{m e s}$ avec $A=\left(\alpha_{i j}\right)$ et $\alpha_{i j}=\left|\left\langle\mid U^{(j)} \|\right\rangle_{i}\right|^{2}$, l'indice $i$ représentant la ième transition choisie et $j=2,4,6$.

Les probabilités d'émission, les durées de vie radiatives des niveaux et les rapports d'embranchement des transitions en sont alors déduites, en écrivant :

$$
\begin{aligned}
& A_{E D}\left(J^{\prime} \rightarrow J\right)=\frac{64 \pi^{4} e^{2}}{3 h\left(2 J^{\prime}+1\right)} \frac{n\left(n^{2}+2\right)^{2}}{9 \overline{\lambda^{3}}} S_{E D}\left(J \rightarrow J^{\prime}\right), \\
& A_{M D}\left(J^{\prime} \rightarrow J\right)=\frac{64 \pi^{4} e^{2}}{3 h\left(2 J^{\prime}+1\right)} \frac{n^{3}}{\lambda^{3}} S_{M D}\left(J \rightarrow J^{\prime}\right), \\
& \frac{1}{\tau_{r}\left(J^{\prime}\right)}=\sum_{J} A\left(J^{\prime} \rightarrow J\right) \text { et } \beta_{r}\left(J^{\prime} \rightarrow J\right)=\frac{A\left(J^{\prime} \rightarrow J\right)}{\sum_{J} A\left(J^{\prime} \rightarrow J\right)},
\end{aligned}
$$

$\operatorname{avec} A\left(J^{\prime} \rightarrow J\right)=A_{E D}\left(J^{\prime} \rightarrow J\right)+A_{M D}\left(J^{\prime} \rightarrow J\right)$.

Notons enfin que dans le cas où $J$ et $J$ ' sont les états fondamental et premier excité, on a aussi, plus simplement :

$$
\frac{1}{\tau_{r}}=\frac{8 \pi n^{2}}{\bar{\lambda}^{2}} \frac{g_{J}}{g_{J^{\prime}}} \int \sigma_{a}(\mathrm{v}) \mathrm{d} \mathrm{v} \quad \text { avec } \quad \frac{g_{J}}{g_{J^{\prime}}}=\frac{2 J+1}{2 J^{\prime}+1} .
$$




\section{CARACTÉRISTIQUES LASER DE DIFFÉRENTS SYSTÈMES}

\subsection{Cristaux dopés $\mathrm{Ce}^{3+}$ pour lasers $\mathrm{UV}$ accordables ou amplificateurs d'impulsions brèves}

Les ions de terres rares trivalents présentent tous des bandes d'absorption très larges dans l'UV et le VUV dues à des transitions optiques $4 f^{\mathrm{n}} \rightarrow 4 f^{\mathrm{n}-1} 5 d$. Suivant la nature des réseaux hôtes, les ions $\mathrm{Ce}^{3+}$, $\operatorname{Pr}^{3+}$ et $\mathrm{Nd}^{3+}[\underline{33}-\underline{38}, 21]$ peuvent ainsi conduire à des bandes d'émission UV très intéressantes dues aux transitions inverses $4 f^{\text {n- }} 5 d \rightarrow 4 f^{n}$. Grâce à leur caractère interconfigurationnel, ces transitions optiques sont en effet permises à l'approximation dipolaire électrique et les sections efficaces d'absorption et d'émission en question sont très élevées, de l'ordre de quelques $10^{-18} \mathrm{~cm}^{2}$. D'autre part, comme le montre la figure $\underline{5}$, les bandes sont non seulement très larges, de quelques dizaines de $\mathrm{nm}$, mais leurs profils comme leurs positions varient aussi de façon importante d'un réseau à l'autre. Dans le cas du $\mathrm{Ce}^{3+}$, par exemple, les deux pics d'émission principaux (Fig. 6) proviennent de transitions optiques vers les deux niveaux spin-orbite ${ }^{2} \mathrm{~F}_{5 / 2}$ et ${ }^{2} \mathrm{~F}_{7 / 2}$ de la configuration électronique $4 f^{1}$, lesquels sont plus ou moins éclatés et mélangés sous l'effet du champ cristallin local.

Nous sommes donc en présence de systèmes lasers vibroniques à 4-niveaux au même titre que les ions de transition du groupe du fer $[\underline{50}, \underline{29}]$ dans $\mathrm{Cr}^{4+}: \mathrm{YAG}_{\mathrm{O}} \mathrm{Ti}^{3+}: \mathrm{Al}_{2} \mathrm{O}_{3}$, lequel est le matériau de base du fameux laser accordable «Titane:saphir» ou Ti:Sa. Cependant, les sections efficaces d'émission $\sigma_{e}$ comme les domaines d'accordabité relatifs $\frac{\Delta E}{E}$ des systèmes dopés terres rares sont plus proches de ceux de lasers à centres colorés tels que $\mathrm{KCl}$ :Tl. En se reportant au tableau $\underline{1}$, on constate en effet que la section efficace d'émission du $\mathrm{Ce}^{3+}$ dans le cristal $\mathrm{LiCaAlF}_{6}(\mathrm{Ce}: \mathrm{LiCAF}$ en abrégé), par exemple, est intermédiaire entre celle du KCL:Tl ${ }^{0}$ et celle du matériau laser infrarouge récemment découvert $\mathrm{Cr}^{2+}: \mathrm{ZnSe}[\underline{56}, \underline{70}]$. Son accordabilité relative $\frac{\Delta E}{E}$ est de l'ordre de $12 \%$, donc plus réduite que celle du $\mathrm{Cr}^{2+}: \mathrm{ZnSe}$ ou du Ti:Sa (laquelle est exceptionnellement élevée grâce à un éclatement Jahn-Teller important de l'état fondamental de l'ion $\mathrm{Ti}^{3+}$ dans $\mathrm{Al}_{2} \mathrm{O}_{3}$ ), mais nettement plus élevée, par exemple, que dans le cas des amplificateurs à verres dopés $\mathrm{Er}^{3+}$ ou $\mathrm{Tm}^{3+}$, les fameux matériaux EDFA et TDFA, actuellement étudiés pour le multiplexage en longueur d'onde des télécommunications par fibres optiques.

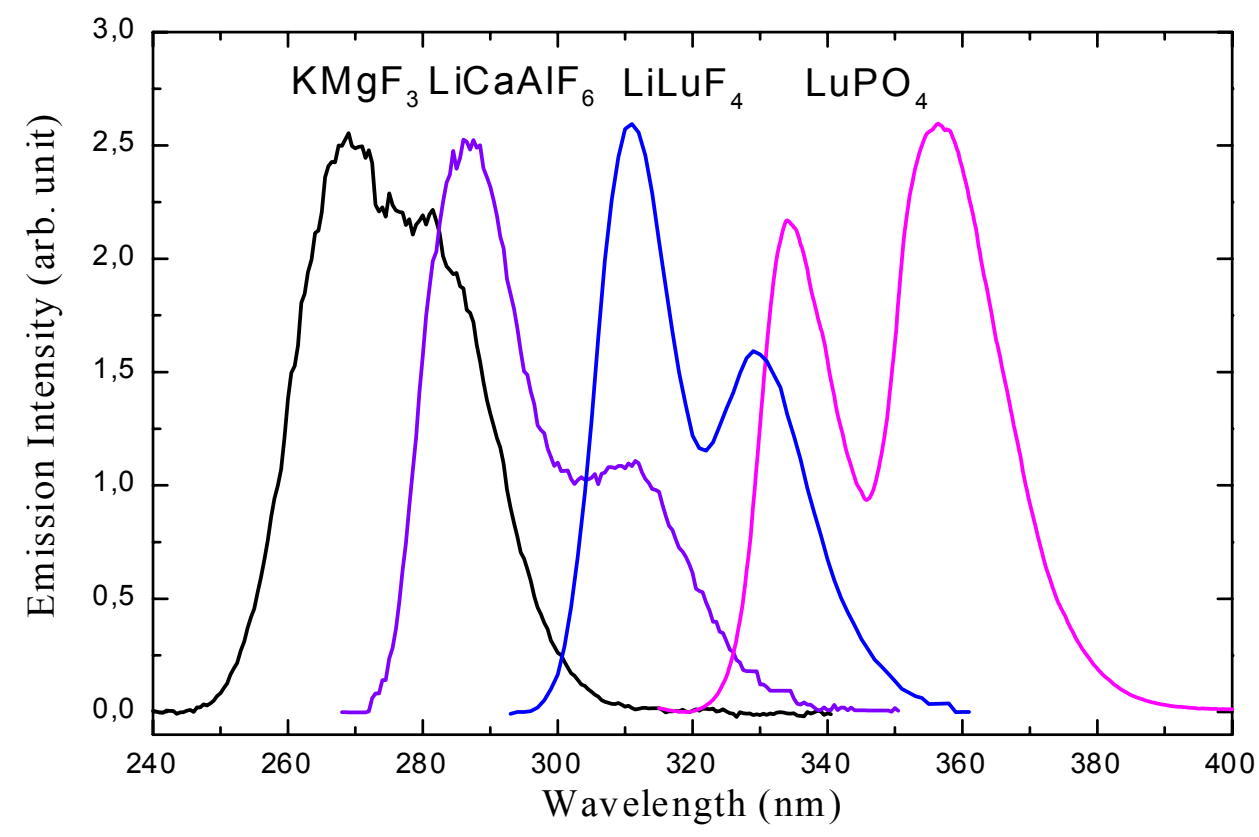

FIG. 5. Spectres d'émission de l'ion $\operatorname{Pr}^{3+}$ dans diverses matrices cristallines. 


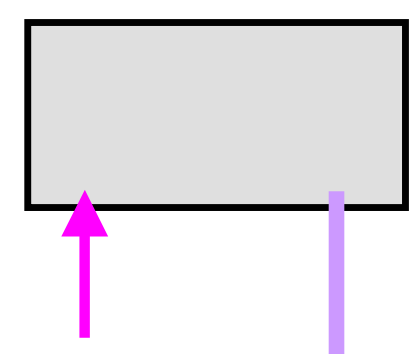

PUMP

$\mathrm{KrF}(248 \mathrm{~nm})$

$\mathrm{XeCl}(308 \mathrm{~nm})$

Nd:YAG $(213,266,355 \mathrm{~nm})$

CVL $(255,271,289 \mathrm{~nm})$

Ce:LiCAF (290 nm)

LASER

)
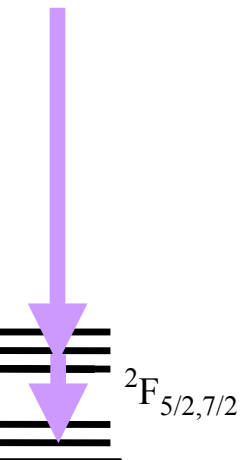

$\mathrm{Ce}^{3+}$

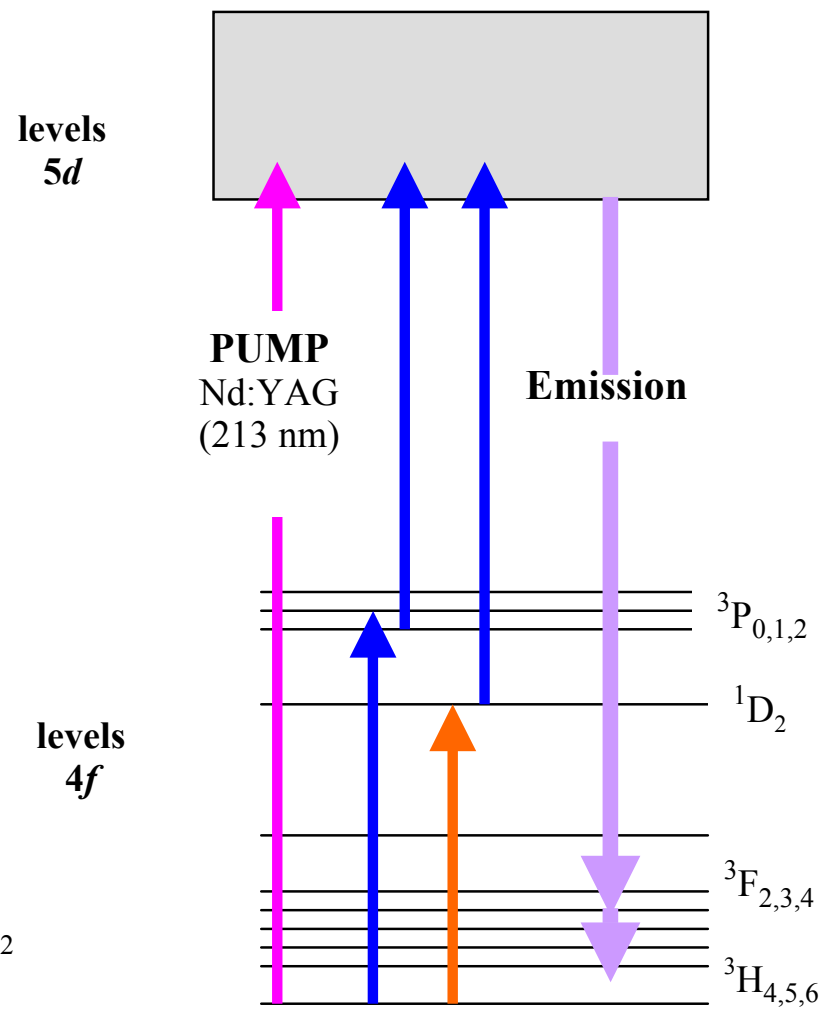

$\mathbf{P r}^{3+}$

FIG 6. Schémas d'excitation/émission des ions $\operatorname{Pr}^{3+}$ et $\mathrm{Ce}^{3+}$.

\begin{tabular}{|c|c|c|c|}
\hline $\begin{array}{c}\text { Systèmes } \\
\text { vibroniques }\end{array}$ & $\lambda(\mathbf{n m})$ & $\begin{array}{c}\sigma_{e m}\left(\mathbf{c m}^{2}\right) \\
\tau_{\mathrm{f}}\end{array}$ & $\Delta \mathbf{E} / \mathbf{E}$ \\
\hline $\begin{array}{c}\mathrm{Ce}^{3+}: \mathrm{LiCaAlF}_{6} \\
(\mathrm{Ce}: \mathrm{LiCAF})\end{array}$ & $280-315$ & $\begin{array}{c}6,8.10^{-18} \\
50 \mathrm{~ns}\end{array}$ & $12 \%$ \\
\hline $\begin{array}{c}\mathrm{Ti}^{3+}: \mathrm{Al}_{2} \mathbf{O}_{3} \\
(\mathrm{Ti}: \mathrm{Sa})\end{array}$ & $700-1000$ & $\begin{array}{l}3.10^{-19} \\
3,2 \mu \mathrm{s}\end{array}$ & $\begin{array}{c}35 \% \\
\text { (effet J.T.) }\end{array}$ \\
\hline KCl:TI ${ }^{0}$ & $1400-1600$ & $\begin{array}{c}1,3.10^{-17} \\
1,6 \mu \mathrm{s}\end{array}$ & $14 \%$ \\
\hline $\mathrm{Cr}^{2+}: \mathrm{ZnSe}$ & $2200-2900$ & $\begin{array}{c}8.10^{-19} \\
8 \mu \mathrm{s}\end{array}$ & $27 \%$ \\
\hline
\end{tabular}

\begin{tabular}{|l|c|c|c|}
\hline EDFA & $1500-1600$ & qqs $10^{-21}$ & $6-7 \%$ \\
TDFA & $1400-1500$ & qqs ms & \\
\hline
\end{tabular}

TAB. 1. Données spectroscopiques des systèmes lasers vibroniques les plus importants et comparaison avec les verres dopés $\mathrm{Er}^{3+}$ et $\mathrm{Tm}^{3+}$, EDFA et TDFA respectivement. 
Les systèmes laser UV large-bande à base d'ions $\mathrm{Ce}^{3+}$ fonctionnent parfaitement en régime impulsionnel $[\underline{15}, \underline{44}, \underline{59}, \underline{63}, \underline{61}, \underline{49}, \underline{35}, \underline{37}, \underline{38}]$, ceci grâce à une fluence de pompage à saturation très

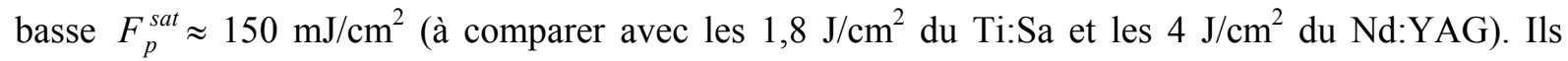
peuvent être pompés par diverses sources d'excitation : lasers à excimères $\mathrm{KrF}(248 \mathrm{~nm})$ et $\mathrm{XeCl}$ (308 nm), laser tout-solide Nd:YAG pompé par diodes, triplé, quadruplé, voire quintuplé en fréquence $(355,266$ et $213 \mathrm{~nm})$, laser à vapeur de cuivre CVL doublé ou mélangé en fréquence $(255,289$ et 271 $\mathrm{nm})$, ou lasers du même type tel que le laser Ce:LiSAF $\left(\mathrm{LiSrAlF}_{6}\right)$ pour pomper un laser Ce:LLF $\left(\mathrm{LiLuF}_{4}\right)$ à $290 \mathrm{~nm}$. Ils peuvent être aisément accordés en longueur d'onde sur une trentaine de $\mathrm{nm}$ tout en gardant une bonne efficacité et une excellente qualité de faisceau dans un domaine de longueur d'onde (Fig. 5) couvert par aucune autre source laser simple et compacte. En outre, ce sont actuellement les seuls matériaux, grâce à leur largeur de bande d'émission d'une dizaine de $\mathrm{THz}$, susceptibles d'amplifier des impulsion brèves sub-picosecondes dans le domaine UV en question.

Les seuls vrais inconvénients de ces systèmes dopés terres rares sont la brièveté de leur temps de vie d'émission (quelques ns), la nécessité d'une croissance cristalline très soignée ainsi que la nécessité de trouver des matériaux à largeur de bande interdite la plus grande possible.

Un temps de vie bref signifie en effet une intensité de pompage à saturation d'autant plus élevée $I_{P}^{s a t} \approx 3000 \mathrm{~kW} / \mathrm{cm}^{2}$ (à comparer avec les $600 \mathrm{~kW} / \mathrm{cm}^{2}$ du Ti:Sa et des $10 \mathrm{~kW} / \mathrm{cm}^{2} \mathrm{du} \mathrm{Nd}: Y A G$ ) ainsi qu'une faible capacité de stockage de l'énergie. Il sera donc difficile, sinon impossible, de faire fonctionner un quelconque système dopé $\mathrm{Ce}^{3+}$ en régime continu.

Une croissance cristalline non optimisée conduit quant à elle à la formation de défauts structuraux ou à l'introduction d'impuretés donnant naissance à des centres pièges absorbants très préjudiciables au fonctionnement laser. Ces centres colorés transitoires ou permanents piègent en effet les électrons optiques qui ont été portés dans la bande de conduction des matériaux hôtes, soit directement, soit par absorption dans l'état excité émetteur le plus bas de la configuration $5 d$, lorsque ces matériaux sont soumis à des rayonnements UV intenses. Il s'agit du phénomène bien connu de solarisation. C'est souvent le cas, par exemple, avec les cristaux de $\mathrm{Ce}^{3+}: \mathrm{LiCaAlF}_{6}$ dans lesquels l'ion trivalent $\mathrm{Ce}^{3+}$ se substitue à un ion divalent $\mathrm{Ca}^{2+}$ en créant un défaut de charge.

Seuls les matériaux à grande bande interdite tels que certains fluorures ont réellement conduit à un fonctionnement laser, ceci précisément pour «transférer» le minimum d'électrons optiques vers les centres pièges par l'intermédiaire de la bande de conduction.

Il s'agit donc de choisir et de fabriquer des matériaux avec des propriétés physico-chimiques bien précises, ce qui réduit évidemment le nombre de possibilités, donc aussi les domaines d'émission accessibles.

Les meilleures performances laser sont obtenues actuellement avec les systèmes Ce:LiCAF et Ce:LiSAF, avec des rendement $\eta_{\text {pente }}$ de l'ordre de 35 à $40 \%$ [44] et Ce:LLF $[\underline{61}, \underline{49}, \underline{35}, \underline{36}, \underline{38}]$ avec des rendements de l'ordre de 40 à $55 \%$. Ce dernier système est certainement le plus intéressant puisque les cristaux de $\mathrm{LiLuF}_{4}$ sont relativement faciles à fabriquer et peuvent être soumis à des fluences laser importantes. Aucun dommage optique ni effet de solarisation n'a été observé par exemple après avoir soumis les cristaux au rayonnement laser à $248 \mathrm{~nm}$ d'un laser à excimère $\mathrm{KrF}$ avec des impulsions allant jusqu'à $4 \mathrm{~J} / \mathrm{cm}^{2} . \mathrm{LiLuF}_{4}$, peut-être à cause d'une fusion congruente, est d'ailleurs moins sujet à la formation de centres colorés que $\mathrm{LiYF}_{4}$.

Des travaux sont actuellement en cours à la fois pour trouver des matrices nouvelles pouvant donner des rayonnements laser complémentaires (c'est le cas de Ce:LuPO $4-c f$. Fig. 5- seul oxyde dans lequel on ait trouvé du gain laser [37]) ainsi que pour remédier aux problèmes de solarisation pouvant apparaître dans les matériaux déjà éprouvés (en particulier, en co-dopant les matériaux avec des ions compensateurs de charge ou servant de centres de recombinaison pour les électrons optiques piégés [6] $]$. 


\subsection{Matériaux dopés $\mathrm{Nd}^{3+}$ : caractéristiques générales et pertes optiques}

Bien que fortement concurrencés par les matériaux dopés par l'ion $\mathrm{Yb}^{3+}$, les matériaux dopés $\mathrm{Nd}^{3+}$ restent encore aujourd'hui et certainement encore pour longtemps les systèmes laser les plus efficaces. Connus plus particulièrement pour leur émission laser 4-niveaux vers $1,06 \mu \mathrm{m}$ (transition ${ }^{4} \mathrm{~F}_{3 / 2} \leftrightarrow$ ${ }^{4} \mathrm{I}_{11 / 2}$ ), ces matériaux sont aussi très utilisés pour leurs émissions laser à 1,32 , voire à $1,44 \mu \mathrm{m}$ (transitions ${ }^{4} \mathrm{~F}_{3 / 2} \leftrightarrow{ }^{4} \mathrm{I}_{13 / 2}$ ), ainsi que pour leur émission quasi 3-niveaux vers $0,94 \mu \mathrm{m}$ (transition ${ }^{4} \mathrm{~F}_{3 / 2} \leftrightarrow{ }^{4} \mathrm{I}_{9 / 2}$ ) (年ig. 7). On les rencontre aussi bien dans les systèmes de très fortes puissances (lasers $\mathrm{Nd}: Y A G$ ou Nd:YAP de plusieurs $\mathrm{kW}$ pour la découpe et le traitement des matériaux ou amplificateurs à verres phosphates ou silicates pour le fusion nucléaire) que dans les systèmes micropuces (lasers pour le micro-usinage ou la télémétrie à sécurité oculaire). Tous se heurtent néanmoins, à des degrés divers, à deux types de problèmes : des pertes d'énergie stockée nonnégligeables dans l'état excité émetteur ${ }^{4} \mathrm{~F}_{3 / 2}$, ce que nous allons développer quelque peu ci-après parce que cela constitue une bonne illustration de ce qui a été exposé précédemment au paragraphe $\underline{2}$, mais aussi une limitation dans la production d'impulsions brèves, ce que nous ne discuterons pas ici, qui est non seulement due à des bandes d'émission relativement étroites mais surtout [11], en particulier pour la transition à $1,06 \mu \mathrm{m}$, à cause d'une durée de vie relativement longue du niveau terminal ${ }^{4} \mathrm{I}_{11 / 2}$ de l'émission laser. Le tableau $\underline{2}$ regroupe les paramètres de fonctionnement des matériaux les plus importants.

Une étude récente portant sur l'amplification d'impulsions courtes de deux types de verres dopés $\mathrm{Nd}^{3+}$ a donc montré $[12,14]$ que des pertes d'énergie importantes pouvaient avoir lieu dans l'état excité émetteur. Ces pertes se manisfestent en particulier par un taux d'inversion nettement plus bas, de l'ordre de $30 \%$, que celui prévu et un taux de désexcitation plus rapide. Une étude spectroscopique approfondie a alors été menée en considérant aussi bien les pertes par absorption dans l'état excité (ESA) du rayonnement de pompage que de celui des rayonnements émis.

\begin{tabular}{|c|c|c|c|c|c|c|}
\hline Matériau & $\begin{array}{c}\alpha_{p}\left(\text { à } \lambda_{p}\right) \\
\text { et } \\
\Delta \lambda_{p}\end{array}$ & $\begin{array}{l}\tau_{R} \\
\text { et } \\
\tau_{F}\end{array}$ & $\begin{array}{c}\sigma_{e m}\left(\grave{a} \lambda_{L}\right) \\
\text { et } \\
\Delta \lambda_{L}\end{array}$ & $\begin{array}{c}\mathrm{K}_{\mathrm{Nd}} \\
\text { max Nd } \\
\text { concentration }\end{array}$ & $\begin{array}{l}\text { Conductivité } \\
\text { thermique } \\
\left(\mathrm{W} / \mathrm{m}^{\circ} \mathrm{K}\right)\end{array}$ & $\begin{array}{c}\text { Expansion } \\
\text { thermique } \\
\left(10^{-6} /{ }^{\circ} \mathrm{K}\right)\end{array}$ \\
\hline $\begin{array}{c}\text { YAG:Nd } \\
\left(\mathrm{Y}_{3} \mathrm{Al}_{5} \mathrm{O}_{12}\right)\end{array}$ & $\begin{array}{c}9 \mathrm{~cm}^{-1} \\
\text { (à } 808 \mathrm{~nm} \text { ) } \\
0.9 \mathrm{~nm} \\
\text { pour } 1,1 \mathrm{at} \% \mathrm{Nd}\end{array}$ & $\begin{array}{c}255 \mu \mathrm{s} \\
\text { et } 220 \mu \mathrm{s} \\
\text { pour } \\
1 \mathrm{at} \% \mathrm{Nd}\end{array}$ & $\begin{array}{l}2,7 \times 10^{-19} \mathrm{~cm}^{2} \\
\text { (à } 1,064 \mu \mathrm{m} \text { ) } \\
\text { et } 0,6 \mathrm{~nm}\end{array}$ & $\begin{array}{c}0,18 \\
1,25 \mathrm{at} \% \mathrm{Nd} \\
\left(1,725 \times 10^{20} \mathrm{~cm}^{-3}\right)\end{array}$ & $10-13$ & 7 \\
\hline $\begin{array}{l}\text { YAP:Nd } \\
\left(\mathrm{YAlO}_{3}\right)\end{array}$ & $\begin{array}{c}11,5 \mathrm{~cm}^{-1} \\
(\text { à } 813 \mathrm{~nm}, \mathrm{E} / / \mathrm{c}) \\
1,6 \mathrm{~nm} \\
\text { pour } 0,77 \mathrm{at} \% \mathrm{Nd}\end{array}$ & $\begin{array}{c}180 \mu \mathrm{s} \\
\text { pour } \\
0,7 \mathrm{at} \% \mathrm{Nd}\end{array}$ & $\begin{array}{c}2.4 \times 10^{-19} \mathrm{~cm}^{2} \\
(\text { à } 1,079 \mu \mathrm{m}, \mathrm{E} / \mathrm{c}) \\
\text { et } 1,6 \mathrm{~nm}\end{array}$ & $\begin{array}{c}0,8-0,95 \\
1,8 \mathrm{at} \% \mathrm{Nd} \\
\left(3,546 \times 10^{20} \mathrm{~cm}^{-3}\right)\end{array}$ & 11 & $\begin{array}{c}9,5(/ / \mathrm{a}) \\
4,3(/ / \mathrm{b}) \\
10,8(/ / \mathrm{c})\end{array}$ \\
\hline $\begin{array}{l}\text { YLF:Nd } \\
\left(\mathrm{YLiF}_{4}\right)\end{array}$ & & $520 \mu \mathrm{s}$ & $\begin{array}{c}1.8 \times 10^{-19} \mathrm{~cm}^{2} \\
(\text { à } 1,0 \mu \mathrm{m}, \mathrm{E} \mathrm{c} \text { ) } \\
\text { et } 1,3 \mathrm{~nm}\end{array}$ & $\begin{array}{c}0,34 \\
2 \mathrm{at} \% \mathrm{Nd}\end{array}$ & 6 & \\
\hline $\mathrm{YVO}_{4}: \mathrm{Nd}$ & $\begin{array}{c}40 \mathrm{~cm}^{-1} \\
(\mathrm{a} 808,5 \mathrm{~nm}) \\
1,7 \mathrm{~nm} \\
\text { pour } 1 \mathrm{at} \% \mathrm{Nd} \\
\end{array}$ & $75-115 \mu \mathrm{s}$ & $\begin{array}{c}6,5 \times 10^{-19} \mathrm{~cm}^{2} \\
(\text { à } 1,064 \mu \mathrm{m}, \mathrm{E} / / \mathrm{c}) \\
\text { et } 0,9 \mathrm{~nm}\end{array}$ & $0,2-0,3$ & 5,2 & $\begin{array}{c}7,3(/ / a) \\
11,4(/ / \mathrm{c})\end{array}$ \\
\hline $\begin{array}{c}\text { KGW:Nd } \\
\left(\mathrm{KGd}\left(\mathrm{WO}_{4}\right)_{2}\right)\end{array}$ & $\begin{array}{c}8 \mathrm{~cm}^{-1} \\
\left(\mathrm{a} 810 \mathrm{~nm}, \mathrm{E} / / \mathrm{n}_{\mathrm{m}}\right) \\
1,2 \mathrm{~nm} \\
\text { pour } 3 \mathrm{at} \% \mathrm{Nd} \\
\end{array}$ & $120 \mu \mathrm{s}$ & $\begin{array}{c}3 \times 10^{-19} \mathrm{~cm}^{2} \\
\left(\text { à } 1,067 \mu \mathrm{m}, \mathrm{E} / / \mathrm{n}_{\mathrm{m}}\right) \\
\text { et } 1,2 \mathrm{~nm}\end{array}$ & $\begin{array}{c}10 \text { at } \% \mathrm{Nd} \\
\left(6,4 \times 10^{20} \mathrm{~cm}^{-3}\right)\end{array}$ & 3,8 & $\begin{array}{c}4 \\
3,6 \\
8,5\end{array}$ \\
\hline $\begin{array}{c}\text { Verre:Nd } \\
\text { Phosphate } \\
\text { Silicate }\end{array}$ & & $330 \mu \mathrm{s}$ & $\begin{array}{l}\sim 3 \times 10^{-20} \mathrm{~cm}^{2} \\
(\text { à } 1,055 \mu \mathrm{m}) \\
\text { et } 20-25 \mathrm{~nm}\end{array}$ & & 1 & 10 \\
\hline
\end{tabular}

TAB. 2. Caractéristiques physiques et spectroscopiques des principales matrices (cristaux et verres) dopées $\mathrm{Nd}^{3+}$. 


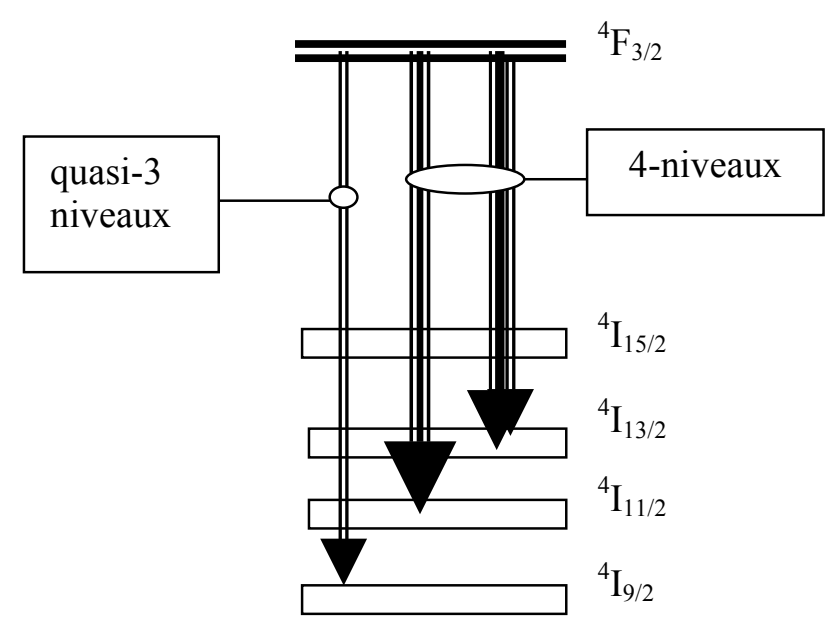

FIG. 7. Principales transitions laser de l'ion $\mathrm{Nd}^{3+}$.
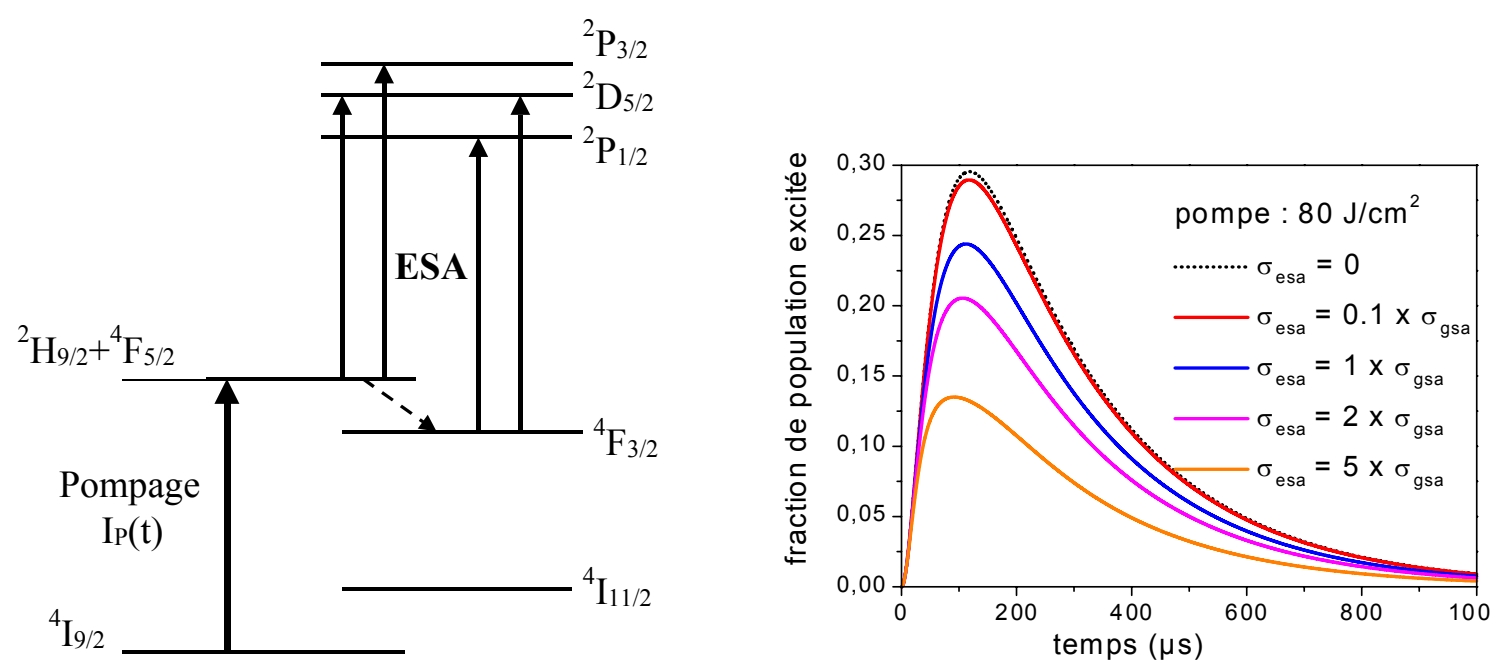

FIG. 8. Mécanismes d'absorption dans l'état excité du rayonnement de pompage optique.
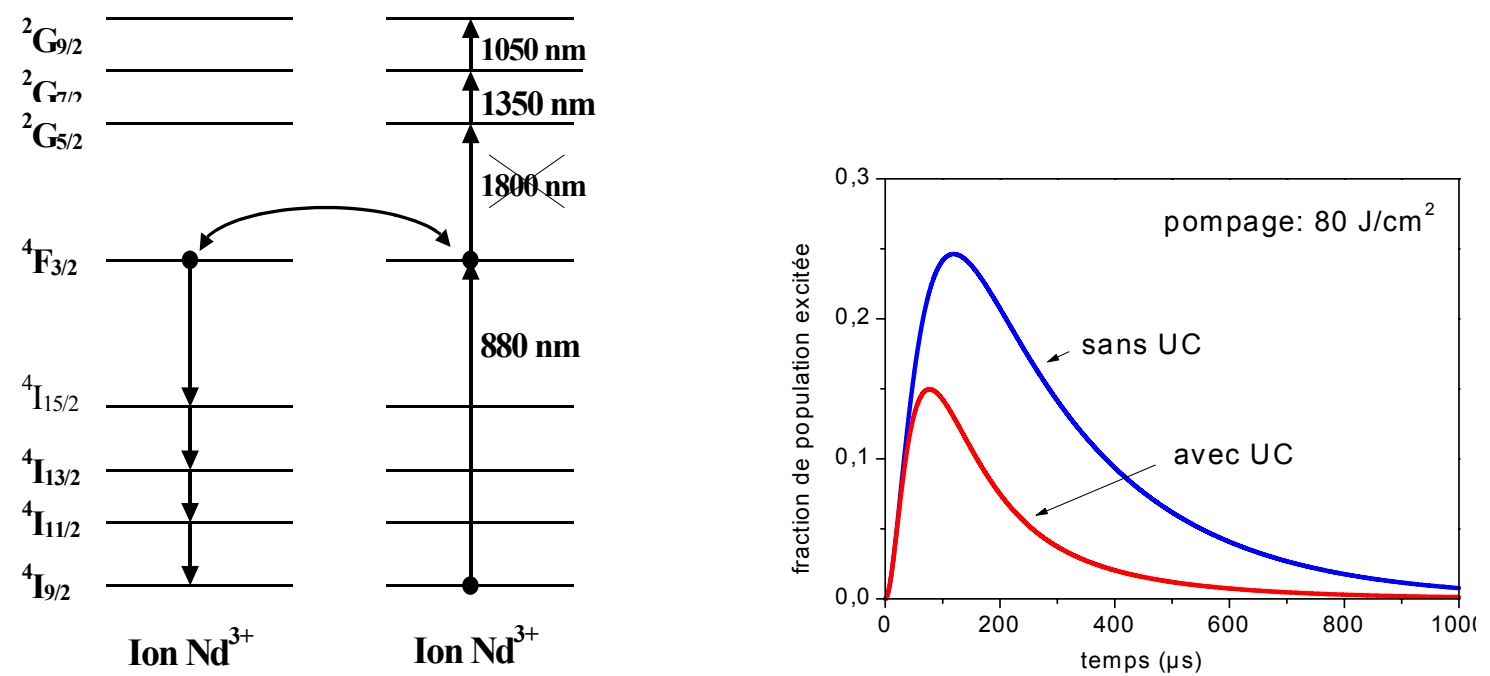

FIG. 9. Mécanismes d'up-conversion (transfert d'énergie + absorption dans l'état excité du rayonnement émis) et de migration de l'énergie d'excitation dans les matériaux dopés $\mathrm{Nd}^{3}$. 
L'influence de l'ESA à la longueur de pompage a été étudiée par l'intermédiaire de la résolution d'une équation numérique analogue à l'équation (17) réécrite sous la forme :

$$
\frac{\partial N_{e}}{\partial t}=\varepsilon_{p} \sigma_{p} N \frac{I_{p}}{h \mathrm{v}_{p}}-\left[\left(\varepsilon_{p} \sigma_{p}+\sigma_{p}^{e s a}\right) \frac{I_{p}}{h \mathrm{v}_{p}}+\frac{1}{\tau_{f}}\right] N_{e} .
$$

En supposant $\varepsilon_{p} \approx 1$, un pompage impulsionnel de la forme $I_{p}(t)=\frac{F_{p}}{\tau_{p}^{2}} t e^{-t / \tau_{p}}$ avec $\tau_{p} \approx 34 \mu$ s et en introduisant, dans le cas d'un verre phosphate de type LG 760 (verre Hoya), les paramètres $N=2,8.10^{20} \mathrm{~cm}^{-3}, \tau_{f}=315 \mu \mathrm{s}, \quad \sigma_{p}=\sigma_{a}(782 \mathrm{~nm})=1,4.10^{-21} \mathrm{~cm}^{2}$, soit $F_{p}^{\text {sat }}=\frac{h \mathrm{v}_{p}}{\sigma_{p}}=180 \mathrm{~J} / \mathrm{cm}^{2}$, on obtient pour $F_{p}=80 \mathrm{~J} / \mathrm{cm}^{2}$, en faisant varier le rapport $\frac{\sigma_{p}^{e s a}}{\sigma_{p}}$ de 0,1 à 5 , les courbes donnant $\beta=\frac{N_{e}}{N}$ de la figure $\underline{8}$. D'après ces courbes, les pertes d'énergie stockée commenceraient à être significatives lorsque $\sigma_{p}^{e s a} \geq \sigma_{p}$. Or, comme le montre la figure $\underline{8}$, il s'agit soit d'ESA dans le niveau émetteur ${ }^{4} \mathrm{~F}_{3 / 2}$ soit d'ESA dans le niveau pompé ${ }^{2} \mathrm{H}_{9 / 2}+{ }^{4} \mathrm{~F}_{5 / 2}$. D'autre part, si on estime le rapport $\frac{\sigma_{p}^{\text {esa }}}{\sigma_{p}}$ à l'aide des spectres d'absorption et du formalisme de Judd-Ofelt, on le trouve inférieur à 0,05 dans le premier cas et inférieur à l'unité dans le second. Mais dans ce dernier, le peuplement thermique du niveau absorbant à la température ambiante est tel que $N\left({ }^{2} \mathrm{H}_{9 / 2},{ }^{4} \mathrm{~F}_{5 / 2}\right) \leq 10^{-2} N\left({ }^{4} \mathrm{~F}_{3 / 2}\right)$. L'ESA à la longueur de pompage ne suffit donc pas à expliquer les pertes observées.

Les pertes d'énergie stockée ont été attribuées en fait presque essentiellement au phénomène d'upconversion (Fig. 9). On le confirme aisément en résolvant l'équation (18) en posant:

$$
\begin{aligned}
& W_{\uparrow} N_{e}=\Gamma_{F D}^{2}+W_{\text {mig }}, \\
& \Gamma_{F D}^{2}=\left(\frac{4}{3}\right)^{2} \pi^{3}\left(C_{1}+C_{2}\right) N_{e}^{2}, \\
& W_{\text {mig }}=\pi\left(\frac{2 \pi}{3}\right)^{5 / 2} \sqrt{\left(C_{1}+C_{2}\right) C_{3}}\left(N-N_{e}\right) N_{e}, \\
& C_{i}=\frac{3 c}{8 \pi^{4} n^{2}} \int \sigma_{e}\left(\lambda_{i}\right) \sigma_{e}^{e s a}\left(\lambda_{i}\right) \mathrm{d} \lambda_{i},
\end{aligned}
$$

avec $i=1$ ou 2 suivant qu'il s'agit de transfert par upconversion du rayonnement émis à $\lambda_{1} \approx 1,06 \mu \mathrm{m}$ ou à $\lambda_{2} \approx 1,32 \mu \mathrm{m}$,

et

$$
C_{3}=\frac{3 c}{8 \pi^{4} n^{2}} \int \sigma_{e}(\lambda) \sigma_{a}(\lambda) \mathrm{d} \lambda
$$

pour tenir compte de la migration de l'énergie à la longueur $\lambda \approx 880 \mathrm{~nm}$.

Les microparamètres de transfert $C_{i}$ étant estimés auparavant après avoir enregistré les spectres de sections efficaces d'absorption dans les états fondamental et excité $\sigma_{a}$ et $\sigma_{e}^{e s a}$ et les spectres de sections efficaces d'émission stimulée $\sigma_{e}$ dans les domaines de longueurs d'onde considérés (Fig. 10), on obtient alors les courbes reportées sur la figure $\underline{9}$, courbes calculées en utilisant les mêmes valeurs de $\varepsilon_{p}, \tau_{p}, N, \tau_{f}, \sigma_{p}$ et $F_{p}$ que précédemment. Ces courbes sont en parfait accord avec les dynamiques de fluorescence observées et elles montrent en effet sans ambiguité que l'upconversion peut être responsable de près de $30 \%$ de perte sur l'énergie stockée. 

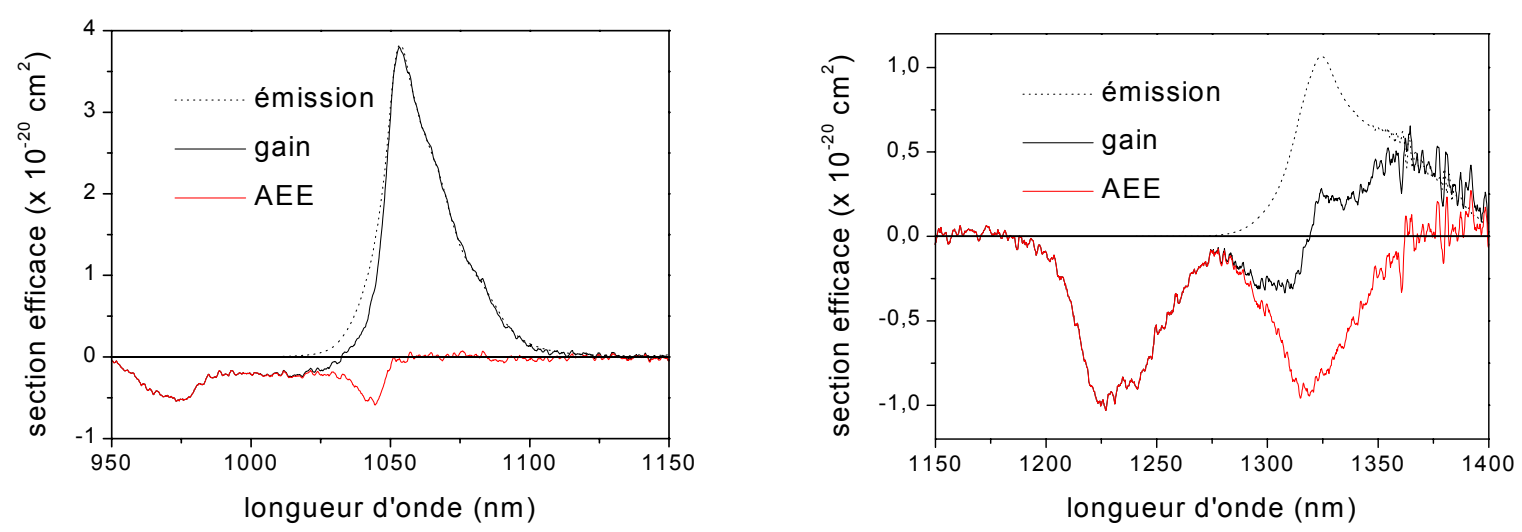

FIG. 10. Spectres d'émission stimulée, d'absorption dans l'état excité et de gain dans un verre phosphate dopé $\mathrm{Nd}^{3+}$.

\subsection{Cristaux à basse énergie de phonons pour lasers moyen-infrarouge}

De nombreux matériaux laser à base d'ions de terres rares peuvent fonctionner dans ce domaine de longueurs d'onde, i.e. entre environ 2 et $5 \mu \mathrm{m}$. Les matériaux dopés $\mathrm{Tm}^{3+}$ et/ou $\mathrm{Ho}^{3+}$ donnent des émissions laser autour de $2 \mu \mathrm{m}[\underline{58}, \underline{19}, \underline{6}]$, ceux qui sont dopés $\mathrm{Er}^{3+}, \mathrm{Ho}^{3+}$ ou $\mathrm{Dy}^{3+}$ des émissions laser autour de 2,8 et $3 \mu \mathrm{m}[72,60,31,26]$, et ceux qui sont dopés $\mathrm{Dy}^{3+}, \mathrm{Er}^{3+} \mathrm{ou}^{3+} \mathrm{Pr}^{3+}$ des émissions laser vers $4,3,4,6$ et $7,2 \mu \mathrm{m}[54,2, \underline{2}, \underline{27}, \underline{4}, \underline{5}, \underline{67}]$. Cependant, seuls ceux à base d'ions $\mathrm{Ho}^{3+}$ et/ou d'ions $\mathrm{Tm}^{3+}$ et ceux à base d' $\mathrm{Er}^{3+}$ émetteurs de rayonnements laser vers 2 et $2,8 \mu \mathrm{m}$, respectivement, présentent actuellement des caractéristiques et des conditions de fonctionnement réellement compétitives avec les autres types de sources laser (OPOs essentiellement). Par conséquent seuls ces systèmes seront discutés dans la suite.

Bien que les systèmes dopés $\mathrm{Ho}^{3+}$ conduisent généralement à des sections efficaces d'émission stimulée plus importantes (donc à des rendements d'extraction plus élevés) avec des valeurs entre 0,3 et $1,8 \cdot 10^{-20} \mathrm{~cm}^{2}$, que les systèmes dopés $\mathrm{Tm}^{3+}$, avec des sections efficaces de 0,1 à $0,5 \cdot 10^{-20} \mathrm{~cm}^{2}$, ces systèmes présentent plusieurs inconvénients : 1) des bandes d'absorption mal adaptées aux longueurs d'onde des diodes laser de puissance actuellement disponibles dans le commerce et pouvant être utilisées comme sources de pompage optique, ce qui signifie la nécessité de codoper les matériaux par d'autres ions tels que les ions $\mathrm{Cr}^{3+}$ (ou $\mathrm{Er}^{3+}$ ) et/ou $\mathrm{Tm}^{3+}$, lesquels absorbent le rayonnement de pompage et le transmettent à l'ion $\mathrm{Ho}^{3+}$ en jouant un rôle de «sensibilisateurs », 2) des pertes par upconversion importantes.

C'est la raison pour laquelle les lasers à $2 \mu \mathrm{m}$ à base $\mathrm{d}^{\prime} \mathrm{Ho}^{3+}$, si étudiés au cours des années 80 et au début des années 90, en particulier pour l'application médicale [53], ont quelque peu cédé le pas aux matériaux (verres et monocristaux) monodopés par des ions $\mathrm{Tm}^{3+}$. Un laser Ho:YAG, néanmoins, a fonctionné récemment de façon très efficace vers $2,1 \mu \mathrm{m}$ en insérant le cristal dans la même cavité qu'un cristal de Tm:YAG pompé par diodes et émettant lui-même à 2,01 $\mu \mathrm{m}$ [23] . la figure 11 :

Les matériaux dopés $\mathrm{Tm}^{3+}$ sont très attrayants pour au moins trois raisons. Comme le montre

1) ils peuvent être directement pompés dans le niveau ${ }^{3} \mathrm{H}_{4}$ à l'aide de diodes laser à $790 \mathrm{~nm}$;

2) à forte concentration d'ions dopants, des transferts d'énergie très efficaces entre ions proches voisins ( « relaxation croisée ») viennent dépeupler le niveau ${ }^{3} \mathrm{H}_{4}$ au profit $\mathrm{du}$ niveau émetteur ${ }^{3} \mathrm{~F}_{4}$;

3) l'émission laser à $2 \mu \mathrm{m}$, à cause d'un couplage électron-phonon relativement important (comme dans le cas de l'ion $\mathrm{Yb}^{3+}[16, \underline{17}, \underline{43}]$ ), est habituellement large ce qui donne la possibilité d'accorder la longueur d'onde laser sur plusieurs dizaines de $\mathrm{nm}$. 


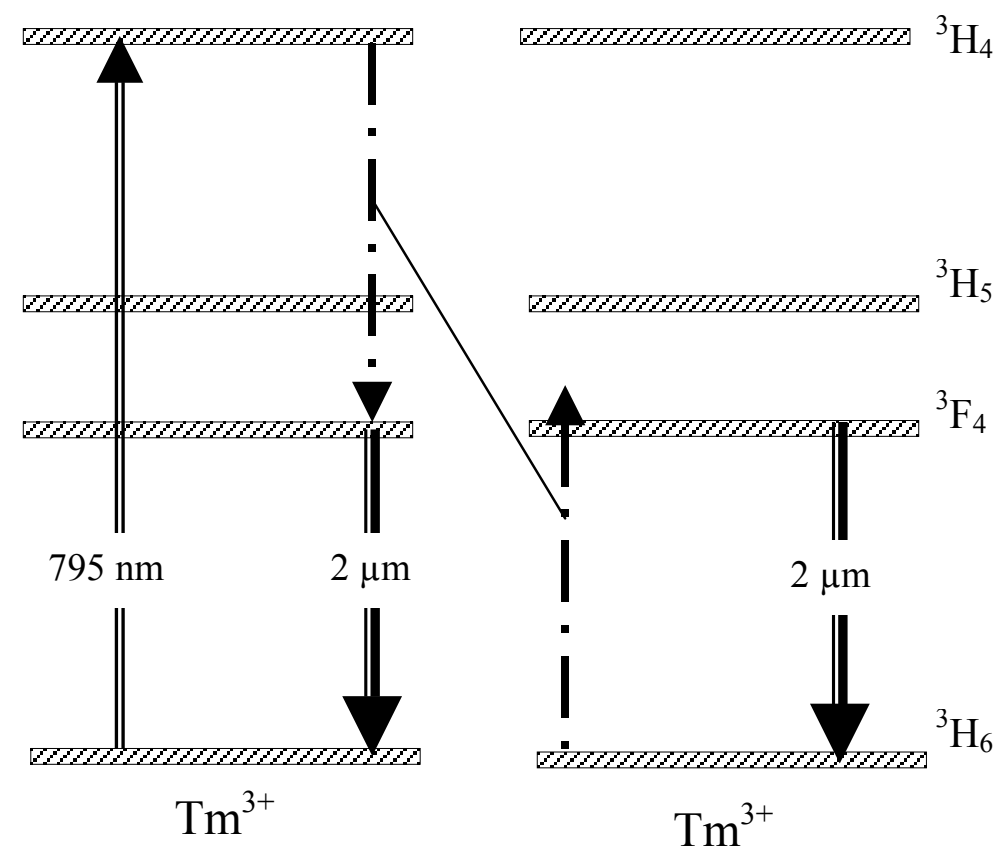

FIG. 11. Schéma de pompage optique et de relaxation croisée utilisés pour le fonctionnement laser à $2 \mu \mathrm{m}$ de l'ion $\mathrm{Tm}^{3+}$.

En principe, dans ces systèmes dopés $\mathrm{Tm}^{3+}$, en raison de la relaxation croisée, chaque photon de pompage optique pourrait conduire à deux photons laser à $2 \mu \mathrm{m}$, ce qui signifierait une efficacité de pompage de 2 . En réalité, cette efficacité de pompage, notée $\varepsilon_{P}$, est donnée par:

$$
\varepsilon_{P}=\frac{W_{n r}+\frac{\beta_{r}}{\tau_{r}}+2 W_{c r}}{W_{n r}+\frac{1}{\tau_{r}}+2 W_{c r}},
$$

où $W_{n r}, \tau_{r}^{-1}$ représentent respectivement les probabilités non-radiative (multiphonons) et radiative des transitions partant du niveau ${ }^{3} \mathrm{H}_{4}$, et $\beta_{r}$ le facteur d'embranchement de la transition ${ }^{3} \mathrm{H}_{4} \rightarrow{ }^{3} \mathrm{~F}_{4} . W_{c r}$ représente la probabilité de transfert d'énergie par relaxation croisée, laquelle dépend approximativement de $N_{\mathrm{Tm}}$, la concentration en ions $\mathrm{Tm}^{3+}$, suivant la relation :

$$
W_{c r} \approx \frac{1}{\tau_{0}}\left[\frac{N_{\mathrm{Tm}}}{N_{0}}\right]^{2},
$$

où $\tau_{0}$ est le temps de vie d'émission à faible concentration d'ions dopants et $N_{0}$ une concentration critique au-delà de laquelle la relaxation croisée domine sur tout autre processus de désexcitation radiatif ou non-radiatif.

Par conséquent, $\varepsilon_{P}$ est toujours inférieur à 2 , soit à cause d'une relaxation non-radiative $W_{n r}$ trop importante, soit à cause d'une relaxation croisée $W_{c r}$ peu efficace. Cette dernière peut être attribuée à une concentration en ions trop basse ou à des interactions ion-ion trop faibles, ce qu'on peut quantifier en mesurant le recouvrement spectral des bandes d'émission et d'absorption ${ }^{3} \mathrm{H}_{4} \rightarrow{ }^{3} \mathrm{~F}_{4}$ et ${ }^{3} \mathrm{H}_{6} \rightarrow{ }^{3} \mathrm{~F}_{4}$, en utilisant des expressions telles que les expressions (무).

Pour toutes ces raisons, bien que des matériaux à grandes énergies de phonon tels que le Tm:YAG donnent d'excellents résultats, plusieurs matériaux à basse énergie de phonon, les fluorures $\mathrm{Tm}: \mathrm{LiYF}_{4}$ et Tm: $\mathrm{KY}_{3} \mathrm{~F}_{10}$ ainsi que l'oxyde $\mathrm{Tm}: \mathrm{Y}_{2} \mathrm{O}_{3}$ (énergies de phonon inférieures à $450 \mathrm{~cm}^{-1}$ ), ont été examinés avec succès ces dernières années $[\underline{7}, \underline{6}, \underline{18}]$. Les fluorures sont particulièrement 
remarquables par le niveau de pertes qu'ils autorisent puisqu'on a pu les faire fonctionner en régime continu avec des pentes de rendement de l'ordre de $50 \%$ et des miroirs de sortie de $40 \%$ de transmission. De plus, ces systèmes fonctionnent préférentiellement vers 1850 et $1900 \mathrm{~nm}$, donc dans des domaines de longueurs d'onde très complémentaires de ceux des oxydes tels que Tm: $\mathrm{Y}_{2} \mathrm{O}_{3}$ et Tm:YAG, lesquels fonctionnent vers 1950 et $2050 \mathrm{~nm}$. Le principal inconvénient de Tm: $\mathrm{Y}_{2} \mathrm{O}_{3}$ est sa croissance cristalline puisqu'il s'agit d'un matériau très réfractaire dont la température de fusion avoisine $2500{ }^{\circ} \mathrm{C}$ et dont seuls des petits cristaux de bonne qualité optique peuvent être obtenus. $\mathrm{Ce}$ système et ses isotypes $\mathrm{Sc}_{2} \mathrm{O}_{3}$ et $\mathrm{Lu}_{2} \mathrm{O}_{3}$, cependant, restent intéressants pour leurs excellentes propriétés thermomécaniques.

En raison d'un pic d'absorption de l'eau très intense autour de 2,9 $\mu \mathrm{m}$, les lasers à solides dopés $\mathrm{Er}^{3+}$ émetteurs de rayonnement dans ce domaine de longueurs d'onde sont également très attrayants et sont utilisés avec succès dans le domaine médical, en particulier en microchirurgie et en ophtalmologie.

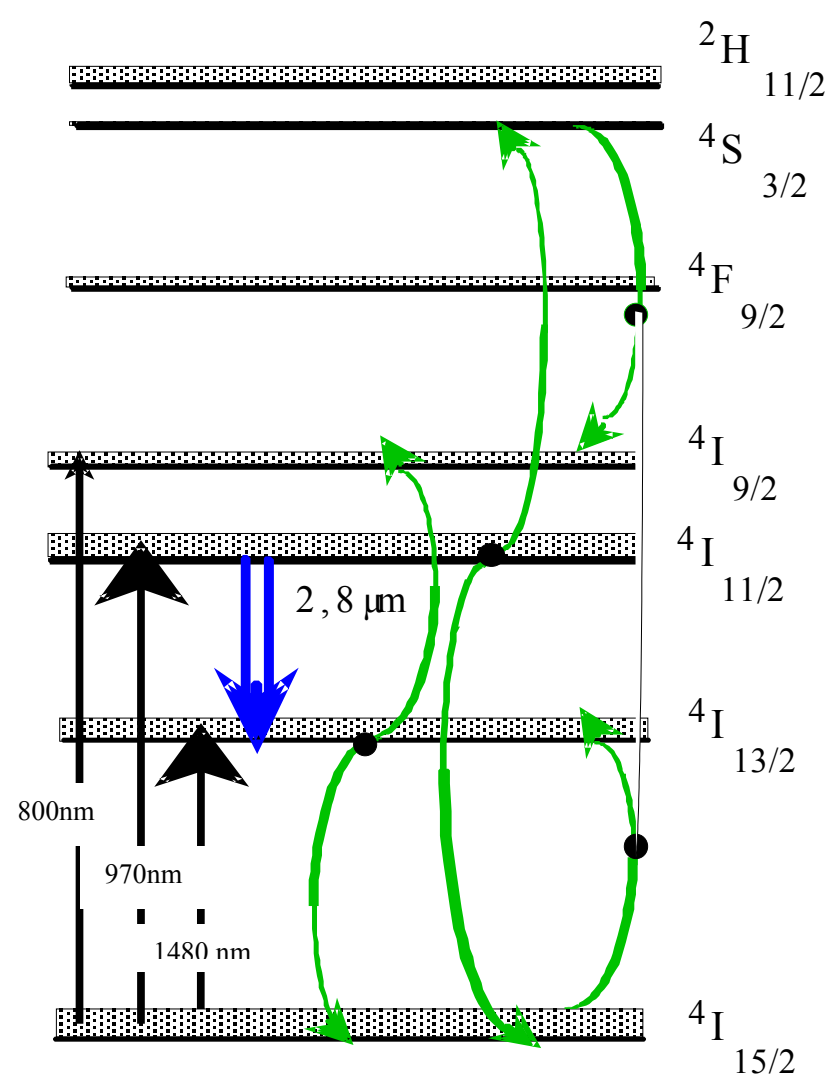

FIG. 12. Schéma de pompage optique et de transferts d'énergie utilisé pour le fonctionnement laser à $2,8 \mu \mathrm{m}$ de l'ion $\mathrm{Er}^{3+}$.

Des produits commerciaux à base de cristaux de YAG et de YSGG $\left(\mathrm{Y}_{3} \mathrm{Sc}_{2} \mathrm{Ga}_{3} \mathrm{O}_{12}\right)$ dopés $\mathrm{Er}^{3+}$ existent déjà sur le marché. Cependant, grâce à des relaxations multiphonons réduites entre niveau émetteur ${ }^{4} \mathrm{I}_{11 / 2}$ et niveau terminal ${ }^{4} \mathrm{I}_{13 / 2}$ de la transition laser (Fig. 12), donc à des rendements quantiques de fluorescence plus élevés, les matériaux hôtes les plus prometteurs sont certainement les fluorures tels que les cristaux $\mathrm{LiYF}_{4}[72, \underline{22}, \underline{25}, \underline{32}], \mathrm{BaY}_{2} \mathrm{~F}_{8}[60]$ et $\mathrm{CaF}_{2}[31, \underline{32}]$ ou le verre ZBLAN $[64,24]$. En effet, comparés aux oxydes, ces fluorures dopés $\mathrm{Er}^{3+}$ conduisent à des spectres d'émission plus larges dans des domaines de longueurs d'onde complémentaires. En outre, comme le montre la figure 12 , on peut les pomper à différentes longueurs d'onde, en particulier avec des diodes autour de 800 et $980 \mathrm{~nm}$ dans les niveaux ${ }^{4} \mathrm{I}_{9 / 2}$ et ${ }^{4} \mathrm{I}_{11 / 2}$, respectivement. On peut même les faire fonctionner, grâce à une combinaison complexe d'ESA, d'upconversion et de relaxation croisée, en pompant vers 1480 $\mathrm{nm}$ dans le niveau terminal ${ }^{4} \mathrm{I}_{15 / 2}$ de la transition laser, ce qui n'est pas banal [32]. Il est nécessaire en tout cas, quelque soit la longueur d'onde d'excitation, d'utiliser des matériaux fortement dopés en ions 
$\mathrm{Er}^{3+}$, d'abord pour absorber suffisamment le rayonnement de pompage mais aussi pour dépeupler efficacement le niveau terminal métastable de la transition laser, par l'intermédiaire de transferts d'énergie par upconversion, et éviter tout phénomène d'embouteillage.

Quand ils sont pompés en continu, ces systèmes laser conduisent ainsi à des rendements nettement plus élevés que les oxydes avec des valeurs de l'ordre de 30 à $50 \%$ et des puissances absorbées au seuil voisines de $50 \mathrm{~mW}$. On peut d'ailleurs remarquer qu'une efficacité de $50 \%$ est plus élevée que l'efficacité de conversion quantique limite donnée par $\eta_{q}=\frac{h v_{e}}{h v_{P}} \approx 35 \%$ dans le cas d'un pompage à $980 \mathrm{~nm}$ et une émission laser à $2,8 \mu \mathrm{m}$. Ce résultat s'interprète précisément par les forts transferts d'énergie par upconversion ayant lieu dans le niveau terminal ${ }^{4} \mathrm{I}_{13 / 2}$ et à la repopulation du niveau émetteur ${ }^{4} \mathrm{I}_{11 / 2}$ via les relaxations multiphonons et les transferts d'énergie par relaxation croisée issus du niveau excité supérieur ${ }^{4} \mathrm{~S}_{3 / 2}$.

Comme le montre la courbe de gain $\sigma_{g}=\beta \sigma_{e}-(1-\beta) \sigma_{a}$ du Er: $\mathrm{CaF}_{2}$ obtenue pour $\beta=0,5$ et reportée sur la figure $\underline{13}$, l'émission laser dans ce matériau a lieu préférentiellement à $2,8 \mu \mathrm{m}$ mais peut être probablement accordée de 2,7 à $2,9 \mu \mathrm{m}$.

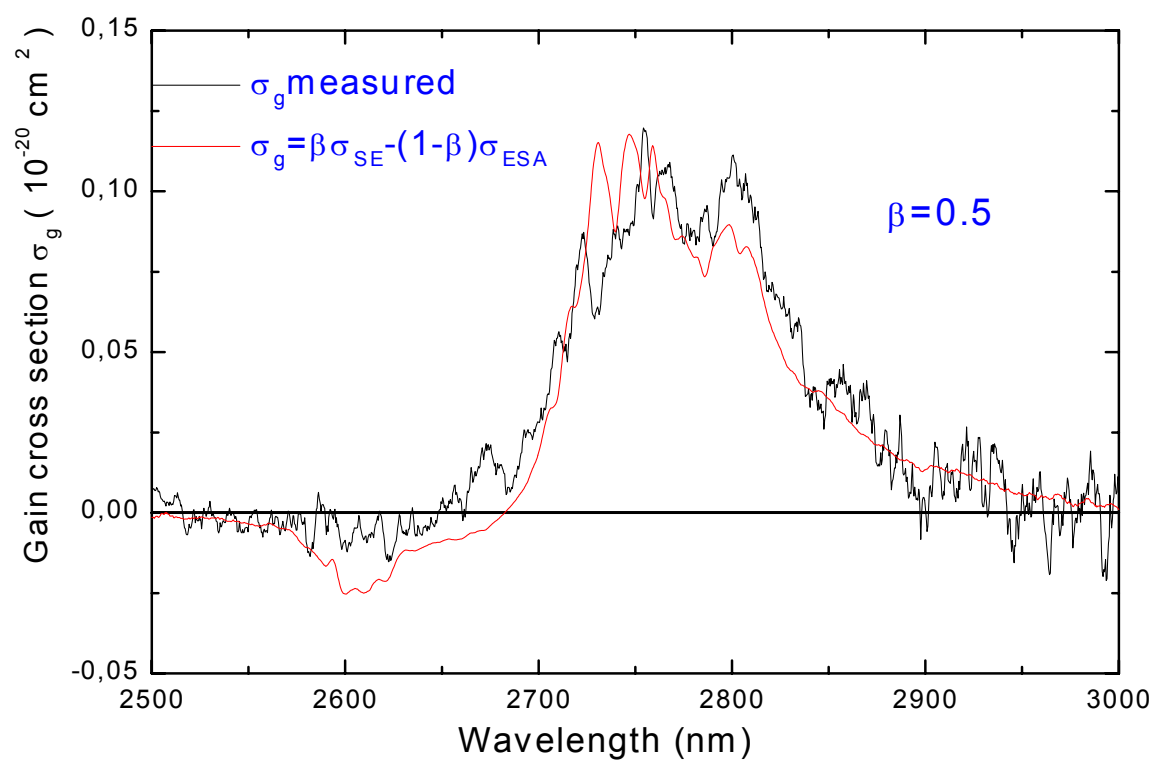

FIG. 13. Spectres de gain mesuré et calculé autour de 2,8 $\mu \mathrm{m}$ dans $\mathrm{CaF}_{2}: \mathrm{Er}^{3+}$.

\section{CONCLUSION}

Après cette présentation rapide, le lecteur réalisera certainement que la caractérisation d'un matériau dopé par un ion de terre n'est pas chose aisée et que de nombreux aspects rentrent en ligne de compte. De fait, de nombreuses pistes restent encore à explorer.

Les cristaux lasers à base d'ions $\mathrm{Ce}^{3+}$ émetteurs de lumière accordables dans l'UV pourront être utilisés, par exemple, pour amplifier des impulsions UV ultra-courtes lesquelles seront produites par d'autres systèmes lasers à solides tels que des sources lasers infrarouges très compactes pompées par diodes, doublées, triplées ou quadruplées en fréquence. Ce type d'applications, néanmoins, dépendra à la fois du niveau de développement des sources et de notre capacité à fabriquer des cristaux de dimension et de qualité optique suffisante. 
Les matériaux dopés par l'ion $\mathrm{Nd}^{3+}$ ont encore vraisemblablement de beaux jours devant eux, dans les micro-systèmes comme dans les systèmes lasers de très fortes puissances. Il est évident, cependant, que les matériaux dopés $\mathrm{Yb}^{3+}$, dont nous n'avons pas parlé dans cette présentation, vont certainement se développer, surtout en tant que systèmes laser à impulsions brèves, en raison de leurs grandes largeurs de gain (résultant d'un couplage ion-réseau plus élevé qu'avec les autres ions terres rares).

L'étude et le développement des sources lasers et amplificateurs à base de matériaux dopés par les ions $\mathrm{Er}^{3+}$ et $\mathrm{Tm}^{3+}$ fonctionnant autour de $1,5 \mu \mathrm{m}$ vont certainement progresser eux-aussi dans les années à venir pour les besoins des télécommunications et des futurs systèmes DWDM, mais aussi de la télémétrie.

Enfin, parmi tous les systèmes fonctionnant dans le moyen-infrarouge, il semble d'ores et déjà évident que les systèmes lasers à $2 \mu \mathrm{m}$ à base de matériaux dopés $\mathrm{Tm}^{3+}$ ou $\mathrm{Ho}^{3+}$, qu'ils soient accordables ou non, à fonctionnement continu ou impulsionnel, vont se développer pour de multiples applications, en tant que sources laser pour la détection LIDAR de " polluants » atmosphériques ou les télécommunications aériennes, par exemple, mais aussi en tant que sources de pompage optique très compactes et très efficaces pour d'autres systèmes laser accordables fonctionnant dans des domaines situés à plus grandes longueurs d'onde (laser $\mathrm{Cr}: \mathrm{ZnSe}$ entre 2,1 et $2,9 \mu \mathrm{m}, \mathrm{OPO} \mathrm{ZnGeP}_{2}$ entre 4 et 8 $\mu \mathrm{m}$, etc...).

Toutes ces possibilités ne pourront pas être pleinement exploitées, en outre, sans des matériaux parfaitement caractérisés mais aussi sans tenir compte des interactions plus ou moins complexes et des mécanismes linéaires et non-linéaires liant la spectroscopie des ions de terres rares sous rayonnement intense et les caractéristiques physiques (effets thermiques, dispersifs,...) et spatiales (cristaux massifs, sous forme de couches minces ou de dimensions nanométriques) des matériaux. 


\section{APPENDICE A : COEFFICIENTS D'EINSTEIN}

Pour des niveaux dégénérés $i$ et $j[\underline{71}]$ de fonctions d'onde $\Psi_{i}$ et $\Psi_{j}$ :

et

$$
\begin{aligned}
& B_{i j}=\frac{1}{g_{i}} \frac{2 \pi q^{2}}{3 h^{2} \varepsilon_{0}} \sum_{k=1}^{g_{i}} \sum_{l=1}^{g_{j}}\left|\left\langle\Psi_{i(k)} / / \vec{r} / / \Psi_{j(l)}\right\rangle\right|^{2}, \\
& A_{j i}=\frac{g_{i}}{g_{j}} B_{i j} \frac{8 \pi h v^{3}}{c^{3}} \\
& B_{i j} g_{i}=B_{j i} g_{j} .
\end{aligned}
$$

\section{APPENDICE B : ÉCLATEMENT D'UN MULTIPLET DE NOMBRE QUANTIQUE $J$ SOUS L'EFFET D'UN CHAMP CRISTALLIN DE SYMÉTRIE DONNÉ}

On voit souvent dans la littérature non spécialisée qu'un multiplet ${ }^{2 \mathrm{~S}+1} \mathrm{~L}_{\mathrm{J}}$ doit éclater sous l'effet $\mathrm{du}$ champ cristallin en $2 J+1$ sous-niveaux Stark dans le cas d'un $J$ entier et en $J+1 / 2$ sous-niveaux (dégénescence de Kramers) dans le cas d'un $J$ demi-entier. Ceci est vrai dans de nombreux cas, en particulier pour des environnements cristallins de basse symétrie. On suppose aussi qu'il en est ainsi dans les systèmes amorphes. Par contre, cela n'est pas aussi "simple» dans le cas de systèmes cristallins de haute symétrie, i.e. cubique, hexagonale ou tétragonale. C'est la raison pour laquelle nous avons réuni dans le tableau suivant le nombre de composantes Stark qu'il fallait attendre dans chacun des cas.

\begin{tabular}{|c|c|c|c|c|c|c|c|c|c|}
\hline$J$ entier & 0 & 1 & 2 & 3 & 4 & 5 & 6 & 7 & 8 \\
\hline Cubique & 1 & 1 & 2 & 3 & 4 & 4 & 6 & 6 & 7 \\
\hline Hexagonale & 1 & 2 & 3 & 5 & 6 & 7 & 9 & 10 & 11 \\
\hline Tetragonale & 1 & 2 & 4 & 5 & 7 & 8 & 10 & 11 & 13 \\
\hline $\begin{array}{c}\text { Plus basses } \\
\text { symétries }\end{array}$ & 1 & 3 & 5 & 7 & 9 & 11 & 13 & 15 & 17 \\
\hline$J$ demi-entier & $1 / 2$ & $3 / 2$ & $5 / 2$ & $7 / 2$ & $9 / 2$ & $11 / 2$ & $13 / 2$ & $15 / 2$ & $17 / 2$ \\
\hline Cubique & 1 & 1 & 2 & 3 & 3 & 4 & 5 & 5 & 6 \\
\hline $\begin{array}{c}\text { Autres } \\
\text { symétries }\end{array}$ & 1 & 2 & 3 & 4 & 5 & 6 & 7 & 8 & 9 \\
\hline
\end{tabular}

\section{APPENDICE C : RELAXATIONS NON-RADIATIVES MULTIPHONONS}

Le temps de vie de fluorescence mesurée $\tau_{f}$ est souvent plus court que le temps de vie radiatif théorique $\tau_{r}$ (que l'on peut déterminer par exemple à partir des spectres d'absorption en utilisant le formalisme de Judd-Ofelt) à cause de relaxations non-radiatives dues au couplage avec les vibrations du réseau. Celles-ci se manifestent alors par la probabilité de relaxation dite "multiphonons » (parce qu'associée à l'émission de plusieurs phonons) notée $W_{n r}$ telle que :

$$
\tau_{f}{ }^{-1}=\tau_{r}{ }^{-1}+W_{n r} .
$$


Cette probabilité est une caractéristique de la matrice et varie en fonction de la température $T$ et l'écart en énergie $\Delta E$ entre le niveau émetteur et le niveau immédiatement inférieur selon la relation suivante [62] :

avec

$$
\begin{gathered}
W_{n r}(T, \Delta E)=B \cdot \exp (-\alpha \cdot \Delta E) \cdot(n(T)+1)^{p}, \\
\alpha=\frac{1}{\hbar \varpi} \cdot\left(\ln \left(\frac{p}{S(n(T)+1)}\right)-1\right) .
\end{gathered}
$$

Dans ce modèle, tous les phonons ont la même énergie $\hbar \varpi, B$ est une constante ne dépendant que de la matrice, $p$ désigne le nombre de phonons mis en jeu, $S$ est le paramètre de Huang-Rhys qui caractérise le couplage électron-phonon (couplage faible dans le cas des ions de terres-rares $\sim 0,01$ ) et $n(T)$ représente le nombre d'occupation des modes de vibration d'énergie $\hbar \varpi$ donnée par la relation de Bose-Einstein :

$$
n(T)=\left(\exp \left(\frac{\hbar \varpi}{k T}\right)-1\right)^{-1}
$$

Pour un écart en énergie $\Delta E$ et une énergie de phonon fixée, la probabilité $W_{n r}$ varie en fonction de la température suivant l'expression :

$$
W_{n r}(T)=W_{0} \cdot(n(T)+1)^{p}=W_{0} \cdot\left(1-\exp \left(-\frac{\hbar \omega}{k T}\right)\right)^{-p},
$$

avec

$$
W_{0}=\mathrm{B} \cdot \exp (-\alpha \cdot \Delta E)
$$

Le couplage $S$ étant très faible, la variation de $\alpha$ avec la température est négligeable et $W_{0}$ est pratiquement indépendant de $T$. D'après l'égalité précédente, on s'aperçoit donc que la probabilité de relaxation $W_{n r}$ augmente quasi-exponentiellement avec la température. Enfin, si l'on se place à une température donnée, par exemple à température ambiante, la relation entre $W_{n r}$ et $\Delta E$, dénommée « loi du gap » est définie par :

$$
W_{n r}(\Delta E)=C \cdot \exp (-\alpha \cdot \Delta E)=C \cdot \exp (-\alpha \cdot p \cdot \hbar \varpi) .
$$

Dans ce cas, les coefficients $C$ et $\alpha$ présentent l'intérêt d'être indépendants de l'ion terre-rare considéré. Pour déterminer leur valeur, il suffit alors de mesurer les durées de vie effectives de plusieurs niveaux émetteurs associés à des écarts $\Delta E$ différents. En général, lorsque le nombre de phonons $p$ mis en jeu dans le processus est inférieur à 4 ou 5 , les transitions non-radiatives multiphonons deviennent importantes et la loi du gap fonctionne assez bien. Elle constitue un bon moyen de comparer les matériaux entre eux suivant le domaine d'émission considéré. 


\section{RÉFÉRENCES BIBLIOGRAPHIQUES}

[1] B.F Aull et H.P. Jenssen, IEEE J. Quant. Electron. QE 18 (1982) 925

[2] N. Barnes et R.E. Allen, IEEE J. Quant. Electron. QE 27 (1991) 277.

[3] M. Bass et M.J. Weber, Laser Focus (sept. 1971) 34-36.

[4] S.R. Bowman, L.B. Shaw, B.J. Feldman et J. Ganem, IEEE J. Quant. Electron. QE 32 (1996) 646.

[5] S.R. Bowman, S.K. Searles, J. Ganem et P. Schmidt, OSA TOPS 26 (1999) 487.

[6] Braud, P.Y. Tigréat, J.L. Doualan et R. Moncorgé, Appl. Phys. B 72 (2001) 909.

[7] P.A. Budni, L.A. Pomeranz, M.L. Lemons, C.A. Miller, J.R. Mosto et E.P. Chicklis, J. Opt. Soc. Am. B 17 (2000) 723.

[8] R. Burlot-Loison, R. Moncorgé, H. Manaa, G. Boulon, Y. Guyot, J. Garcia-Solé et D. CochetMuchy, Opt. Mater. 6 (1996) 313.

[9] W.T. Carnall, P.R. Fields et B.G. Wybourne, J. Chem. Phys. 42 (1965) 3797.

[10] W.T. Carnall, P.R. Fields et K. Rajnak, J. Chem. Phys. 49 (1968) 4412 ; 49 (1968) 4424.

[11] L.D. DeLoach, S.A. Payne, L.L. Chase, L.K. Smith, W.L. Kway et W.F. Krupke, IEEE J. Quant. Electron. QE 29 (1993) 1179.

[12] D. Descamps, Thèse de Doctorat, Université de Paris VI, France (1997).

[13] N. Djeu, V.E. Hartwell, A.A. Kaminskii et A.V. Butashin, Opt. Lett. 22 (1997) 997.

[14] J.L. Doualan, C. Maunier, D. Descamps, J. Landais et R. Moncorgé, Phys. Rev. B 62 (2000) 4459.

[15] M.A. Dubinskii, V.V. Semashko, A.K. Naumov, R. Yu. Abdulsabirov et S.L. Korableva, Laser Physics 3 (1993) 216.

[16] Ellens, H. Andres, A. Meijerink et G. Blasse, Phys. Rev. B 55 (1997) 173.

[17] Ellens, H. Andres, M.L.H. ter Heerdt, R.T. Wegh, A. Meijerink et G. Blasse, Phys. Rev. B 55 (1997) 180.

[18] F.S. Ermeneux, Thèse de Doctorat, Université de Lyon I, France (1999).

[19] F.S. Ermeneux, Y. Sun, R.L. Cone, R.W. Equall, R.L. Hutcheson et R. Moncorgé, OSA TOPS 26 (1999) 476.

[20] L.M. Frantz et J.S. Nodvik, J. Appl. Phys. 34 (1963) 2346.

[21] Y. Guyot, S. Guy et M.F. Joubert, J. Alloys and Comp. 323/324 (2001) 722.

[22] C.E. Hamilton, R.J. Beach, S.B. Sutton, L.H. Furu et W.K. Krupke, Opt. Lett. 19 (1994) 1627.

[23] R.A. Hayward, W.A. Clarkson et D.C. Hanna, in Advanced Solid State Lasers 2000, Tech. Digest paper MB8, p 45.

[24] S.D. Jackson, T.A King et M. Pollnau, Opt. Lett. 24 (1999) 1133.

[25] T. Jensen, A. Diening, G. Huber et B.H.T. Chai, Opt. Lett. 21 (1996) 585.

[26] L.F. Johnson et H.J. Guggenheim, Appl. Phys. Lett. 23 (1973) 96.

[27] S.M. Kirkpatrick, S.R. Bowman, L.B. Shaw et S. Searles, OSA TOPS 19 (1998) 534.

[28] W.F. Krupke, M.D. Shinn, J.E. Marion, J.A. Caird et S.E. Stokowski, J. Opt. Soc. Am. B 3 (1986) 102.

[29] S. Kuck, Appl. Phys. B 72 (2001) 515.

[30] Y.K. Kuo, M.F. Huang et M. Birnbaum, IEEE J. Quant. Electron. QE 31 (1995) 657.

[31] C. Labbé, J.L. Doualan, S. Girard, R. Moncorgé et M. Thuau, in Proceed. Int. Conf. Lasers'98, édité par V.J. Corcoran et TA. Goldman (STS Press, VA, USA, 1999) p. 217.

[32] C. Labbé, J.L. Doualan, S. Girard, R. Moncorgé et M. Thuau, J. Phys. Cond. Matter 12 (2000) 6943.

[33] M. Laroche, A. Braud, S. Girard, J.L. Doualan, R. Moncorgé, M. Thuau et L.D. Merkle, J. Opt. Soc. Am. B 16 (1999) 2269.

[34] M. Laroche, J.L. Doualan, S. Girard, J. Margerie et R. Moncorgé, J. Opt. Soc. Am. B 17 (2000) 1291.

[35] M. Laroche, S. Girard, R. Moncorgé, G.J. Quarles et J.Y. Gesland, in Conf. Digest, CLEO'Europ, Nice, France, 2000 (2000) paper CtuF2, p. 69.

[36] M. Laroche, S. Girard, R. Moncorgé, M. Bettinelli, K. Shimamura et T. Fukuda, in Conf. Digest, CLEO'Europ - EQEC, Munich, Germany (2001) p. 55.

[37] M. Laroche, Thèse de Doctorat, Université de Caen, France (2001).

[38] M. Laroche, S. Girard et R. Moncorgé, J. Opt. Soc. Am. B (2002, à paraître). 
[39] Ph. Le Boulanger, J.L. Doualan, S. Girard, J. Margerie et R. Moncorgé, Phys. Rev. B 60 (1999) 11380 .

[40] M. Lenévé, Thèse de Doctorat, Université de Grenoble, France (1996).

[41] Lowenthal et J.M. Eggleston, IEEE J. Quant. Electron. QE 22 (1986) 1165.

[42] H. Manaa, Y. Guyot et R. Moncorgé, Phys. Rev. B 48 (1993) 3633.

[43] J. Margerie, communication privée.

[44] C.D. Marshall, J.E. Speth, S.A. Payne, W.K. Krupke, G.J. Quarles, V. Castillo et B.H.T. Chai, J. Opt. Soc. Am. B 11 (1994) 2054.

[45] W.E. Martin et D. Milam, IEEE J. Quant. Electron. QE 18 (1982) 1155.

[46] C. Maunier, J.L. Doualan, G. Aka, E. Antic-Fidancev, R. Moncorgé et D. Vivien, Opt. Commun. 184 (2000) 209.

[47] C. Maunier, J.L. Doualan, R. Moncorgé, A. Speghini, M. Bettinelli et E. Cavalli, OSA Tops on Advanced Solid State Lasers 50 (2001) 261.

[48] D.E. McCumber, Phys. Rev. A 136 (1964) 954.

[49] A.J. McGonigle, S. Girard, D.W. Coutts et R. Moncorgé, Electron. Lett. 35 (1999) 1640.

[50] R. Moncorgé, H. Manaa et B. Boulon, Opt. Mater. 4 (1994) 139.

[51] R. Moncorgé, B. Chambon, J.Y. Rivoire, N. Garnier, E. Descroix, P. Laporte, H Guillet, S. Roy, J. Mareschal, D. Pelenc, J. Doury et P. Farge, Opt. Mater. 8 (1997) 109.

[52] P.F. Moulton, J. Opt. Soc. Am. B 3 (1986) 125.

[53] P.F. Moulton et S. Wright, Laser Focus World (march 1992) 65.

[54] M.C. Nostrand, R.H. Page, W.F. Krupke, P.G. Schuneman et L.I. Isaenko, OSA Tops on Advanced Solid State Lasers 26 (1999) 441 ; Opt. Lett. 24 (1999) 1215.

[55] M.C. Nostrand et R.H. Page, OSA Tops on Advanced Solid State Lasers 50 (2001) 438.

[56] R.H. Page, K.I. Shaffers, L.D. DeLoach, G.D. Wilke, F.D. Patel, J.B. Jr. Tassano, S.A. Payne, W.K. Krupke, K.T. Chen et A. Burger, IEEE J. Quant. Electron. QE 33 (1997) 609.

[57] S.A. Payne, J.A. Caird, L.L. Chase, L.K. Smith et W.K. Krupke. J. Opt. Soc. Am. B 8 (1991) 726.

[58] S.A. Payne, L.L. Chase, L.K. Smith et W.L. Kway, IEEE J. Quant. Electron. QE 28 (1992) 2619.

[59] J.F. Pinto, L. Esterowitz et G.J. Quarles, Electron. Lett. 31 (1995) 2009.

[60] M. Pollnau, W. Luthy, H.P. Weber, T. Jensen, G. Huber, A. Cassanho, H.P. Jenssen et R.M. McFarlane, Opt. Lett. 21 (1996) 48.

[61] P. Rambaldi, R. Moncorgé, J.P. Wolf, C. Pédrini et J.Y. Gesland, Opt. Comm., 163 (1998) 146 ; Laser Focus World 33 (1997) 26.

[62] L.A. Riseberg et M.J. Weber, Phys. Rev. 174 (1968) 429.

[63] N. Sarukura, M.A. Dubinskii, Z. Liu, V.A. Semashko, A.K. Naumov, S.L. Korableva, R.Y. Abdulsabirov, K. Edamatsu, Y. Suzuki, T. Itoh et Y. Segawa, IEEE J. Selected Topics Quant. Electron. 1 (1995) 792.

[64] T. Sandrock, A. Diening et G. Huber, Opt. Lett. 24 (1999) 382.

[65] V. Scherbitsky, S. Girard, M. Fromager, R. Moncorgé, N.V. Kuleshov, V.I. Levchenko, V.N. Yakimovich et B. Ferrand, Appl. Phys. B 74 (2002) 367.

[66] V. Semashko , M. Dubinskii, R.Y. Abdulsabirov, S.L. Karobleva, A.K. Naumov, A.S. Nizamutdinov, M.S. Zhuchkov, in SPIE Proceed. of XI Int. Feofilov Symp., Kazan, Russia, 2001 4766 (2002).

[67] L.B. Shaw, S.R. Bowman, B.J. Feldman, et J. Ganem, IEEE J. Quant. Electron. QE 32 (1996) 2166.

[68] M.D. Shinn, F.P. Milanovich, et J.N. Roe, CLEO (Baltimore, USA) April 1989.

[69] J.C. Souriau, C. Borel, Ch. Wyon, C. Li et R. Moncorgé, J. Lumin. 59 (1994) 349 et références citées.

[70] G.J. Wagner, T.J. Carrig, R.H. Page, K.I. Schaffers, J.O. Ndap, X. Ma et A. Butger, Opt. Lett. 24 (1999) 19.

[71] M. Weissbluth, Atoms and Molecules. Academic Press, New York (1978).

[72] C. Wyss , W. Luthy, H.P. Weber, P. Rogin et J. Hulliger, Opt. Comm. 139 (1997) 215. 